01

\title{
Эллипсоидальное включение с оболочкой в анизотропной среде с однородным приложенным электрическим полем
}

\author{
(С) И.В. Лавров, В.Б. Яковлев \\ Национальный исследовательский университет Московский институт электронной техники, \\ 124498 Москва, Зеленоград, Россия \\ e-mail: iglavr@mail.ru
}

(Поступило в Редакцию 16 ноября 2017 г.)

Получено решение электростатической задачи для диэлектрического включения, состоящего из анизотропных ядра и оболочки, помещенного в однородную анизотропную диэлектрическую среду (матрицу) с приложенным однородным электрическим полем. Внешние границы ядра и оболочки считаются эллипсоидами, являющимися софокусными после линейного неортогонального преобразования, устраняющего анизотропию диэлектрических свойств оболочки. Найдены аналитические выражения для потенциала и напряженности электрического поля в матрице, оболочке и ядре, а также выражение для тензора поляризуемости включения. Рассмотрен специальный случай включения с изотропной оболочкой. Полученные выражения применены для случая анизотропного шара с изотропной оболочкой в анизотропной среде. Также показано, что в предельном случае однородного эллипсоидального включения в анизотропной среде полученный результат согласуется с известными решениями.

DOI: $10.21883 / J T F .2018 .10 .46490 .2565$

\section{Введение}

Необходимость в вычислении распределения электрического поля внутри и снаружи включения, погруженного в анизотропную среду, возникает при прогнозировании макроскопических свойств неоднородных текстурированных материалов, т.е. материалов, обладающих анизотропией эффективных характеристик. В частности, при применении метода самосогласованного решения $[1,2]$, называемого также методом эффективной среды, идея которого принадлежит Бруггеману [3], реальная неоднородная среда, окружающая включение, заменяется на однородную с эффективными материальными свойствами.

Вариант обобщения метода эффективной среды на материалы с неоднородными включениями предложен в [4]; для его применения в анизотропном случае требуется знание распределения электрического поля внутри так называемого „эффективного рассеивателя“, погруженного в эффективную анизотропную среду. В работе [5] предложено обобщенное приближение эффективного поля для вычисления макроскопических характеристик неоднородных сред, содержащих неоднородные включения, состоящие из однородного ядра и однородной оболочки, внешние границы которых считаются эллипсоидальными. Для его применения требуется знание связи между средними значениями напряженности поля в ядре и оболочке изолированного включения, погруженного в однородную, возможно, анизотропную среду, и эффективным полем в данной среде.

Настоящая работа является продолжением [6], и ее результаты естественным образом обобщают результаты работы [6] на случай эллипсоидального включения с оболочкой, помещенного в бесконечную анизотропную среду (матрицу) с однородным приложенным электрическим полем. Внешние границы ядра и оболочки включения считаются эллипсоидальными, становящимися софокусными после линейного преобразования, устраняющего анизотропию материальных свойств оболочки. На первый взгляд, это условие является искусственным и не имеющим отношение к реальным ситуациям, однако в реальных неоднородных средах даже эллипсоидальная форма включений в точности практически никогда не встречается, а является модельным приближением для частиц неизометричной формы. По мнению авторов, введение дополнительной связи между формами ядра и оболочки и тензором материального свойства оболочки оправдано, поскольку позволяет получить компактное аналитическое выражение для решения задачи.

В настоящей работе исходная задача для включения в анизотропной среде решается путем сведения ее посредством линейного неортогонального преобразования к задаче для включения с оболочкой в вакууме, решение которой известно [6]. Затем с помощью обратного преобразования из решения задачи в вакууме получается решение исходной задачи в анизотропной среде. Получено также выражение для тензора поляризуемости включения. Рассмотрен частный случай включения с изотропной оболочкой, а также примеры применения полученных результатов для сферического включения с изотропной оболочкой в анизотропной, а затем в изотропной среде. Также рассмотрен предельный случай эллипсоидального однородного включения в анизотропной среде; показано, что результаты в этом случае совпадают с известными $[7,8]$.

Сделаем замечание, касающееся используемых в работе терминов. В математике термин „эллипсоид“ обозначает замкнутую поверхность 2-го порядка, а специаль- 
ного термина, обозначающего тело, ограниченное этой поверхностью, нет. Между тем в физической литературе как в классических трудах [9-11], так и в ряде относительно новых работ $[8,12-14]$ термин ,эллипсоид“ используется для обозначения объемного тела, ограниченного поверхностью 2-го порядка - эллипсоидом. В настоящей работе в согласии с терминологией, принятой в физической литературе, термин „эллипсоид“ будет использоваться для обозначения тела, ограниченного замкнутой поверхностью 2-го порядка, а для обозначения самой этой поверхности будет использоваться термин „поверхность-эллипсоид“.

\section{Постановка задачи и сведение ее к задаче для включения с оболочкой в вакууме}

Рассмотрим диэлектрическое включение, состоящее из ядра, занимающего область $V_{2}$, и оболочки, занимающей область $V_{1}$, помещенное в бесконечную диэлектрическую среду (матрицу) с внешним приложенным однородным электрическим полем напряженностью $\mathbf{E}_{0}$. Область, занимаемую всем неоднородным включением, обозначим как $V$. Материалы, составляющие матрицу, оболочку и ядро, предполагаются однородными и анизотропными с тензорами диэлектрической проницаемости $\varepsilon_{m}, \varepsilon_{1}$ и $\varepsilon_{2}$ соответственно. Граница $S_{2}$ ядра и внешняя граница $S_{1}$ оболочки считаются поверхностямиэллипсоидами, становящимися софокусными после линейного неортогонального преобразования, устраняющего анизотропию диэлектрических свойств оболочки [6]. Ставится задача найти распределение потенциала $\varphi(\mathbf{r})$ и напряженности $\mathbf{E}(\mathbf{r})=-\nabla \varphi(\mathbf{r})$ электростатического поля в ядре и оболочке данного включения, а также в матрице; область, занимаемую матрицей, обозначим как $V_{m}$. Предполагается, что в данной системе свободные заряды отсутствуют.

Математическая формулировка соответствующей краевой задачи для потенциала $\varphi(\mathbf{r})$ имеет вид

$$
\begin{gathered}
\nabla \cdot \varepsilon_{m} \nabla \varphi_{m}(\mathbf{r})=0, \quad \mathbf{r} \in V_{m}, \\
\nabla \cdot \varepsilon_{1} \nabla \varphi_{1}(\mathbf{r})=0, \quad \mathbf{r} \in V_{1}, \\
\nabla \cdot \varepsilon_{2} \nabla \varphi_{2}(\mathbf{r})=0, \quad \mathbf{r} \in V_{2}, \\
\varphi_{m}(\mathbf{r})=\varphi_{1}(\mathbf{r}), \quad\left(\boldsymbol{\varepsilon}_{m} \mathbf{E}_{m}\right)_{n}=\left(\boldsymbol{\varepsilon}_{1} \mathbf{E}_{1}\right)_{n}, \quad \mathbf{r} \in S_{1}, \\
\varphi_{1}(\mathbf{r})=\varphi_{2}(\mathbf{r}), \quad\left(\varepsilon_{1} \mathbf{E}_{1}\right)_{n}=\left(\boldsymbol{\varepsilon}_{2} \mathbf{E}_{2}\right)_{n}, \quad \mathbf{r} \in S_{2}, \\
\left.\varphi_{m}\right|_{\infty}=-\left(\mathbf{E}_{0}, \mathbf{r}\right),
\end{gathered}
$$

где $\varphi_{m}(\mathbf{r}), \mathbf{E}_{m}, \varphi_{1}(\mathbf{r}), \mathbf{E}_{1}$ и $\varphi_{2}(\mathbf{r}), \mathbf{E}_{2}-$ скалярные потенциалы и напряженности электрического поля в матрице, оболочке и ядре соответственно; $\mathbf{n}-$ вектор единичной нормали к соответствующей поверхности; $\nabla-$ векторный дифференциальный оператор Гамильтона, имеющий в исходных декартовых координатах $x^{1}, x^{2}, x^{3}$ вид $\boldsymbol{\nabla}=\mathbf{i} \frac{\partial}{\partial x^{1}}+\mathbf{j} \frac{\partial}{\partial x^{2}}+\mathbf{k} \frac{\partial}{\partial x^{3}}$. Условия (1)-(3) - это уравнения в частных производных, которым должен удовлетворять потенциал $\varphi(\mathbf{r})$ в матрице, оболочке и ядре соответственно. Условия (4) и (5) - это непрерывность $\varphi(\mathbf{r})$ и нормальной составляющей вектора электрической индукции $\mathbf{D}(\mathbf{r})$ на границе оболочки и матрицы и на границе ядра и оболочки соответственно. Условие (6) означает, что на бесконечном удалении от включения потенциал $\varphi(\mathbf{r})$ равен потенциалу приложенного поля $\varphi_{0}=-\left(\mathbf{E}_{0}, \mathbf{r}\right)$. Подразумевается также ограниченность потенциала внутри частицы, вытекающая из физического смысла задачи. Полуоси поверхностейэллипсоидов $S_{i}, i=1,2$ считаются известными и равными $a_{1}^{(i)}, a_{2}^{(i)}, a_{3}^{(i)}$.

Сведем задачу (1)-(6) к аналогичной задаче для включения с оболочкой в вакууме; для этого сделаем линейное неортогональное преобразование координат $\left(\mathbf{r}=\left(x^{1} x^{2} x^{3}\right)^{T}, \mathbf{r}^{\prime}=\left(x^{1^{\prime}} x^{2^{\prime}} x^{3^{\prime}}\right)^{T}\right)$

$$
\mathbf{r}=\mathbf{T}_{m} \mathbf{r}^{\prime}
$$

подобранное таким образом, чтобы (1) преобразовалось к уравнению Лапласа:

$$
\nabla \cdot \varepsilon_{m} \nabla \varphi_{m}(\mathbf{r})=0, \mathbf{r} \in V_{m} \Leftrightarrow \nabla^{\prime} \cdot \nabla^{\prime} \varphi_{m}\left(\mathbf{T}_{m} \mathbf{r}^{\prime}\right)=0, \mathbf{r}^{\prime} \in V_{m}^{\prime},
$$

где $V_{m}^{\prime}$ - область, занимаемая матрицей в системе координат $x^{1^{\prime}} x^{2^{\prime}} x^{3^{\prime}}$. Операторы Гамильтона в системах $x^{1} x^{2} x^{3}$ и $x^{1^{\prime}} x^{2^{\prime}} x^{3^{\prime}}$ связаны друг с другом по формуле

$$
\nabla=\left(\mathbf{T}_{m}^{-1}\right)^{T} \nabla^{\prime}
$$

а связь тензора $\varepsilon_{m}$ с матрицей преобразования (8) имеет вид:

$$
\boldsymbol{\varepsilon}_{m}=\mathbf{T}_{m} \mathbf{T}_{m}^{T} \text {. }
$$

Условно говоря, преобразование (7) устраняет анизотропию диэлектрических свойств матрицы; его также можно рассматривать как преобразование пространства, при этом поверхности-эллипсоиды $S_{1}, S_{2}$, преобразуются соответственно в поверхности-эллипсоиды $S_{1}^{\prime}, S_{2}^{\prime}$, полуоси которых обозначим как $a_{1^{\prime}}^{(i)}, a_{2^{\prime}}^{(i)}, a_{3^{\prime}}^{(i)}, i=1,2$. С целью единственным образом определить преобразование (7) потребуем, наряду с (9), чтобы оси системы координат $x^{1^{\prime}} x^{2^{\prime}} x^{3^{\prime}}$ были направлены вдоль осей поверхности $S_{1}^{\prime}$ с таким соответствием, чтобы ее полуоси были упорядочены: $a_{1^{\prime}}^{(1)}>a_{2^{\prime}}^{(1)}>a_{3^{\prime}}^{(1)}$. Аналогично [6] введем для краткости обозначения для произведений полуосей:

$$
\bar{a}^{(i)}=a_{1}^{(i)} a_{2}^{(i)} a_{3}^{(i)}, \quad \bar{a}^{\prime(i)}=a_{1^{\prime}}^{(i)} a_{2^{\prime}}^{(i)} a_{3^{\prime}}^{(i)}, \quad i=1,2 .
$$

После преобразования (7) условия (1)-(3) примут вид:

$$
\begin{array}{cc}
\nabla^{\prime} \cdot \nabla^{\prime} \varphi_{m}\left(\mathbf{T}_{m} \mathbf{r}^{\prime}\right)=0, & \mathbf{r}^{\prime} \in V_{m}^{\prime}, \\
\nabla^{\prime} \cdot \varepsilon_{1}^{\prime} \nabla^{\prime} \varphi_{1}\left(\mathbf{T}_{m} \mathbf{r}^{\prime}\right)=0, & \mathbf{r}^{\prime} \in V_{1}^{\prime}, \\
\nabla^{\prime} \cdot \varepsilon_{2}^{\prime} \nabla^{\prime} \varphi_{2}\left(\mathbf{T}_{m} \mathbf{r}^{\prime}\right)=0, & \mathbf{r}^{\prime} \in V_{2}^{\prime},
\end{array}
$$

где $V_{1}^{\prime}, V_{2}^{\prime}$ - образы областей $V_{1}, V_{2}$ при преобразовании (7);

$$
\varepsilon_{1}^{\prime}=\mathbf{T}_{m}^{-1} \varepsilon_{1}\left(\mathbf{T}_{m}^{-1}\right)^{T}, \quad \varepsilon_{2}^{\prime}=\mathbf{T}_{m}^{-1} \varepsilon_{2}\left(\mathbf{T}_{m}^{-1}\right)^{T}([15]) .
$$


Образ области $V$, занимаемой всем включением, обозначим как $V^{\prime}$.

Пусть $f_{1}(\mathbf{r})=0$ и $f_{2}(\mathbf{r})=0-$ уравнения поверхностей $S_{1}, S_{2}$, тогда

$$
\left.\mathbf{n}\right|_{S_{i}}=\left.\nabla f_{i}\left|\nabla f_{i}\right|^{-1}\right|_{S_{i}}, \quad i=1,2,
$$

и вторые из этих условий (4), (5) можно переписать в виде

$$
\begin{array}{ll}
\left(\nabla f_{1}, \boldsymbol{\varepsilon}_{m} \mathbf{E}_{m}\right)=\left(\nabla f_{1}, \boldsymbol{\varepsilon}_{1} \mathbf{E}_{1}\right), & \mathbf{r} \in S_{1}, \\
\left(\boldsymbol{\nabla} f_{2}, \boldsymbol{\varepsilon}_{1} \mathbf{E}_{1}\right)=\left(\nabla f_{2}, \boldsymbol{\varepsilon}_{2} \mathbf{E}_{2}\right), & \mathbf{r} \in S_{2} .
\end{array}
$$

Напряженность электрического поля, согласно (8), преобразуется по формулам

$$
\mathbf{E}=\left(\mathbf{T}_{m}^{-1}\right)^{T} \mathbf{E}^{\prime}, \quad \mathbf{E}^{\prime}=\mathbf{T}_{m}^{T} \mathbf{E} .
$$

Запишем равенства (15) в системе координат $x^{1^{\prime}} x^{2^{\prime}} x^{3^{\prime}}$. Преобразуем левую часть первого из этих равенств с учетом (8), (9), (16)

$$
\begin{aligned}
& \left(\nabla f_{1}, \boldsymbol{\varepsilon}_{m} \mathbf{E}_{m}\right)=\left(\left(\mathbf{T}_{m}^{-1}\right)^{T} \boldsymbol{\nabla}^{\prime} f_{1}, \boldsymbol{\varepsilon}_{m}\left(\mathbf{T}_{m}^{-1}\right)^{T} \mathbf{T}_{m}^{T} \mathbf{E}_{m}\right) \\
& \quad=\left(\boldsymbol{\nabla}^{\prime} f_{1},\left(\mathbf{T}_{m}^{-1}\right) \boldsymbol{\varepsilon}_{m}\left(\mathbf{T}_{m}^{-1}\right)^{T} \mathbf{E}_{m}^{\prime}\right)=\left(\boldsymbol{\nabla}^{\prime} f_{1}, \mathbf{E}_{m}^{\prime}\right) .
\end{aligned}
$$

Аналогично для правой части первого равенства с учетом (14) имеем

$$
\left(\nabla f_{1}, \varepsilon_{1} \mathbf{E}_{1}\right)=\left(\nabla^{\prime} f_{1}, \boldsymbol{\varepsilon}_{1}^{\prime} \mathbf{E}_{1}^{\prime}\right)
$$

поэтому первое из равенств (15) в системе $x^{1^{\prime}} x^{2^{\prime}} x^{3^{\prime}}$ принимает вид

$$
\left(\nabla^{\prime} f_{1}, \mathbf{E}_{m}^{\prime}\right)=\left(\nabla^{\prime} f_{1}, \varepsilon_{1}^{\prime} \mathbf{E}_{1}^{\prime}\right), \quad \mathbf{r}^{\prime} \in S_{1}^{\prime}
$$

Для второго из равенств (15) аналогичным образом получим вид в системе $x^{1^{\prime}} x^{2^{\prime}} x^{3^{\prime}}$ :

$$
\left(\nabla^{\prime} f_{2}, \varepsilon_{1}^{\prime} \mathbf{E}_{1}^{\prime}\right)=\left(\nabla^{\prime} f_{2}, \varepsilon_{2}^{\prime} \mathbf{E}_{2}^{\prime}\right), \quad \mathbf{r}^{\prime} \in S_{2}^{\prime}
$$

Первые из условий (4), (5) в системе $x^{1^{\prime}} x^{2^{\prime}} x^{3^{\prime}}$ можно записать в следующей форме:

$$
\begin{aligned}
& \varphi_{m}^{\prime}\left(\mathbf{r}^{\prime}\right)=\varphi_{1}^{\prime}\left(\mathbf{r}^{\prime}\right), \quad \mathbf{r}^{\prime} \in S_{1}^{\prime}, \\
& \varphi_{1}^{\prime}\left(\mathbf{r}^{\prime}\right)=\varphi_{2}^{\prime}\left(\mathbf{r}^{\prime}\right), \quad \mathbf{r}^{\prime} \in S_{2}^{\prime},
\end{aligned}
$$

где $\quad \varphi_{m}^{\prime}\left(\mathbf{r}^{\prime}\right)=\varphi_{m}\left(\mathbf{T}_{m} \mathbf{r}^{\prime}\right), \quad \varphi_{1}^{\prime}\left(\mathbf{r}^{\prime}\right)=\varphi_{1}\left(\mathbf{T}_{m} \mathbf{r}^{\prime}\right), \quad \varphi_{2}^{\prime}\left(\mathbf{r}^{\prime}\right)=$ $=\varphi_{2}\left(\mathbf{T}_{m} \mathbf{r}^{\prime}\right)$. Условие (6) в системе $x^{1^{\prime}} x^{2^{\prime}} x^{3^{\prime}}$ примет вид

$$
\left.\varphi_{m}^{\prime}\right|_{\infty}=-\left(\mathbf{E}_{0}^{\prime}, \mathbf{r}^{\prime}\right)
$$

Таким образом, задача $(1)-(6)$ в системе координат $x^{1^{\prime}} x^{2^{\prime}} x^{3^{\prime}}$ сводится к системе условий $(11)-(13)$, $(17)-(21)$. Получившаяся задача - это задача для эллипсоидального включения с оболочкой, помещенного в вакуум с однородным приложенным полем. Чтобы показать это, перепишем ее формулировку в координатном виде (подразумевается суммирование по повторяющимся индексам):

$$
\begin{gathered}
\nabla_{k^{\prime}} \delta^{k^{\prime} l^{\prime}} \nabla_{l^{\prime}} \varphi_{m}^{\prime}\left(\mathbf{r}^{\prime}\right)=0, \quad \mathbf{r}^{\prime} \in V_{m}^{\prime}, \\
\nabla_{k^{\prime}} \varepsilon_{1}^{k^{\prime} l^{\prime}} \nabla_{l^{\prime}} \varphi_{1}^{\prime}\left(\mathbf{r}^{\prime}\right)=0, \quad \mathbf{r}^{\prime} \in V_{1}^{\prime}, \\
\nabla_{k^{\prime}} \varepsilon_{2}^{k^{\prime} l^{\prime}} \nabla_{l^{\prime}} \varphi_{2}^{\prime}\left(\mathbf{r}^{\prime}\right)=0, \quad \mathbf{r}^{\prime} \in V_{2}^{\prime}, \\
\varphi_{m}^{\prime}\left(\mathbf{r}^{\prime}\right)=\varphi_{1}^{\prime}\left(\mathbf{r}^{\prime}\right), \quad \nabla_{k^{\prime}} f_{1} \delta^{k^{\prime} l^{\prime}}\left(E_{m}\right)_{l^{\prime}}=\nabla_{k^{\prime}} f_{1} \varepsilon_{1}^{k^{\prime} l^{\prime}}\left(E_{1}\right)_{l^{\prime}}, \\
\mathbf{r}_{1}^{\prime} \in S_{1}^{\prime}, \\
\left.\mathbf{r}^{\prime}\right)=\varphi_{2}^{\prime}\left(\mathbf{r}^{\prime}\right), \quad \nabla_{k^{\prime}} f_{2} \varepsilon_{1}^{k^{\prime} l^{\prime}}\left(E_{1}\right)_{l^{\prime}}=\nabla_{k^{\prime}} f_{2} \varepsilon_{2}^{k^{\prime} l^{\prime}}\left(E_{2}\right)_{l^{\prime}} \\
\mathbf{r}_{m}^{\prime} \in S_{2}^{\prime}, \\
\varphi_{\infty}^{\prime}=-\left(E_{0}\right)_{k^{\prime}} x^{k^{\prime}}
\end{gathered}
$$

Координатная форма (22)-(27) задачи в системе $x^{1^{\prime}} x^{2^{\prime}} x^{3^{\prime}}$ не зависит от метрики в этой системе, поэтому можно абстрагироваться от исходной метрики в системе $x^{1} x^{2} x^{3}$ и считать, что метрика в системе $x^{1^{\prime}} x^{2^{\prime}} x^{3^{\prime}}-$ декартова. Тогда вторые из условий (25), (26) равносильны условиям непрерывности нормальной составляющей электрической индукции на поверхностях $S_{1}^{\prime}, S_{2}^{\prime}$, поэтому задача (22) - (27) - это задача для включения с оболочкой, помещенного в вакуум с однородным приложенным полем $\mathbf{E}_{0}^{\prime}$. Диэлектрические проницаемости оболочки и ядра включения равны $\varepsilon_{1}^{\prime}$ и $\varepsilon_{2}^{\prime}$ соответственно.

\section{Решение задачи для включения с оболочкой в вакууме}

Решение задачи $(22)-(27)$ известно и имеет вид [6]

$$
\begin{gathered}
\varphi_{m}^{\prime}\left(\mathbf{r}^{\prime}\right)=\left(\left(-\mathbf{I}+3\left(\bar{a}^{\prime(1)}\right)^{-1} \mathbf{N}^{\prime}\left(\xi^{\prime}\right) \boldsymbol{\alpha}^{\prime}\right) \mathbf{E}_{0}^{\prime}, \mathbf{r}^{\prime}\right) \\
\mathbf{r}^{\prime} \in V_{m}^{\prime}, \quad \xi^{\prime} \geq 0 \\
\varphi_{1}^{\prime}\left(\mathbf{r}^{\prime}\right)=\left(\left(\boldsymbol{\beta}^{\prime(1)}+\mathbf{N}_{0}^{\prime \prime}\left(\xi^{\prime \prime}\right) \boldsymbol{\alpha}^{\prime(1)}\right) \mathbf{E}_{0}^{\prime}, \mathbf{r}^{\prime}\right) \\
\mathbf{r}^{\prime} \in V_{1}^{\prime}, \quad 0 \leq \xi^{\prime \prime} \leq t^{\prime \prime} \\
\varphi_{2}^{\prime}\left(\mathbf{r}^{\prime}\right)=-\left(\mathbf{E}^{\prime(2)}, \mathbf{r}^{\prime}\right)
\end{gathered}
$$

где

$$
\begin{aligned}
\boldsymbol{\alpha}^{\prime}= & 3^{-1} \bar{a}^{\prime(1)}\left[\left(\boldsymbol{\varepsilon}_{1}^{\prime}-\mathbf{I}\right)\left(\mathbf{I}+\left(\mathbf{L}_{0}^{\prime \prime(2)}-v^{\prime} \mathbf{L}_{0}^{\prime \prime(1)}\right)\left(\boldsymbol{\varepsilon}_{2}^{\prime}-\boldsymbol{\varepsilon}_{1}^{\prime}\right)\right)\right. \\
& \left.+v^{\prime}\left(\boldsymbol{\varepsilon}_{2}^{\prime}-\boldsymbol{\varepsilon}_{1}^{\prime}\right)\right] \boldsymbol{\lambda}_{20}^{\prime}, \\
\boldsymbol{\alpha}^{\prime(1)}= & \left(\boldsymbol{\varepsilon}_{2}^{\prime}-\boldsymbol{\varepsilon}_{1}^{\prime}\right) \boldsymbol{\lambda}_{20}^{\prime}, \quad \boldsymbol{\beta}^{\prime(1)}=-\left(\mathbf{I}+\mathbf{L}_{0}^{\prime \prime(2)}\left(\boldsymbol{\varepsilon}_{2}^{\prime}-\boldsymbol{\varepsilon}_{1}^{\prime}\right)\right) \boldsymbol{\lambda}_{20}^{\prime}, \\
\lambda_{20}^{\prime}= & {\left[\left(\mathbf{I}+\mathbf{L}^{\prime(1)}\left(\boldsymbol{\varepsilon}_{1}^{\prime}-\mathbf{I}\right)\right)\left(\mathbf{I}+\left(\mathbf{L}_{0}^{\prime \prime(2)}-v^{\prime} \mathbf{L}_{0}^{\prime \prime(1)}\right)\left(\boldsymbol{\varepsilon}_{2}^{\prime}-\boldsymbol{\varepsilon}_{1}^{\prime}\right)\right)\right.} \\
& \left.+v^{\prime} \mathbf{L}^{\prime(1)}\left(\boldsymbol{\varepsilon}_{2}^{\prime}-\boldsymbol{\varepsilon}_{1}^{\prime}\right)\right]^{-1} .
\end{aligned}
$$$$
\mathbf{E}^{\prime(2)}=\lambda_{20}^{\prime} \mathbf{E}_{0}^{\prime},
$$ 
В первом из выражений (28) $\xi^{\prime}-$ первая из эллипсоидальных координат $\xi^{\prime}, \eta^{\prime}, \xi^{\prime}$, связанных с координатами $x^{1^{\prime}} x^{2^{\prime}} x^{3^{\prime}}$ по формулам

$$
\begin{gathered}
\left(x^{i^{\prime}}\right)^{2}=\frac{\left(\xi^{\prime}+\left(a_{i^{\prime}}^{(1)}\right)^{2}\right)\left(\eta^{\prime}+\left(a_{i^{\prime}}^{(1)}\right)^{2}\right)\left(\xi^{\prime}+\left(a_{i^{\prime}}^{(1)}\right)^{2}\right)}{\left(\left(a_{j^{\prime}}^{(1)}\right)^{2}-\left(a_{i^{\prime}}^{(1)}\right)^{2}\right)\left(\left(a_{k^{\prime}}^{(1)}\right)^{2}-\left(a_{i^{\prime}}^{(1)}\right)^{2}\right)}, \\
i^{\prime}=1^{\prime}, 2^{\prime}, 3^{\prime} \quad\left(i^{\prime} \neq j^{\prime} \neq k^{\prime} \neq i^{\prime}\right), \\
-\left(a_{1^{\prime}}^{(1)}\right)^{2}<\xi^{\prime}<-\left(a_{2^{\prime}}^{(1)}\right)^{2}<\eta^{\prime}<-\left(a_{3^{\prime}}^{(1)}\right)^{2}<\xi^{\prime}<+\infty ;
\end{gathered}
$$

$\mathbf{N}^{\prime}\left(\xi^{\prime}\right)$ - тензорная функция координаты $\xi^{\prime}$ :

$$
\begin{gathered}
\mathbf{N}^{\prime}\left(\xi^{\prime}\right)=\left\|\begin{array}{ccc}
N_{1^{\prime}}^{\prime}\left(\xi^{\prime}\right) & 0 & 0 \\
0 & N_{2^{\prime}}^{\prime}\left(\xi^{\prime}\right) & 0 \\
0 & 0 & N_{3^{\prime}}^{\prime}\left(\xi^{\prime}\right)
\end{array}\right\|, \\
N_{i^{\prime}}^{\prime}\left(\xi^{\prime}\right)=\frac{\bar{a}^{\prime(1)}}{2} \int_{\xi^{\prime}}^{+\infty} \frac{d u}{\left[u+\left(a_{i^{\prime}}^{(1)}\right)^{2}\right] R_{u}^{\prime(1)}}, \quad i^{\prime}=1^{\prime}, 2^{\prime}, 3^{\prime}, \\
R_{u}^{(1)}=\left[\left(u+\left(a_{1^{\prime}}^{(1)}\right)^{2}\right)\left(u+\left(a_{2^{\prime}}^{(1)}\right)^{2}\right)\left(u+\left(a_{3^{\prime}}^{(1)}\right)^{2}\right)\right]^{1 / 2}
\end{gathered}
$$

Во втором из выражений (28) $\xi^{\prime \prime}$ - первая из эллипсоидальных координат $\xi^{\prime \prime}, \eta^{\prime \prime}, \xi^{\prime \prime}$, связанных с координатами $x^{1^{\prime \prime}}, x^{2^{\prime \prime}}, x^{3^{\prime \prime}}$, которые вводятся с помощью преобразования $\left(\mathbf{r}^{\prime \prime}=\left(x^{1^{\prime \prime}} x^{2^{\prime \prime}} x^{3^{\prime \prime}}\right)^{T}\right)$

$$
\mathbf{r}^{\prime}=\mathbf{T}_{1}^{\prime} \mathbf{r}^{\prime \prime}
$$

устраняющего анизотропию оболочки после преобразования (7). Его связь с тензором $\varepsilon_{1}^{\prime}$ имеет вид

$$
\varepsilon_{1}^{\prime}=\mathbf{T}_{1}^{\prime} \mathbf{T}_{\mathbf{1}}^{\prime \mathbf{T}} \text {. }
$$

Образами поверхностей-эллипсоидов $S_{1}^{\prime}, S_{2}^{\prime}$, при преобразовании (35) являются поверхности-эллипсоиды $S_{1}^{\prime \prime}, S_{2}^{\prime \prime}$, которые предполагаются софокусными; их полуоси, обозначенные как $a_{1^{\prime \prime}}^{(i)}, a_{2^{\prime \prime}}^{(i)}, a_{3^{\prime \prime}}^{(i)}(i=1,2)$, связаны соотношениями

$$
\left(a_{k^{\prime \prime}}^{(1)}\right)^{2}=\left(a_{k^{\prime \prime}}^{(2)}\right)^{2}+t^{\prime \prime}, \quad k^{\prime \prime}=1^{\prime \prime}, 2^{\prime \prime}, 3^{\prime \prime},
$$

где $t^{\prime \prime}>0-$ некоторое число. Координаты $\xi^{\prime \prime}, \eta^{\prime \prime}, \xi^{\prime \prime}$ вводятся формулами

$$
\begin{gathered}
\left(x^{i^{\prime \prime}}\right)^{2}=\frac{\left(\xi^{\prime \prime}+\left(a_{i^{\prime \prime}}^{(2)}\right)^{2}\right)\left(\eta^{\prime \prime}+\left(a_{i^{\prime \prime}}^{(2)}\right)^{2}\right)\left(\xi^{\prime \prime}+\left(a_{i^{\prime \prime}}^{(2)}\right)^{2}\right)}{\left(\left(a_{j^{\prime \prime}}^{(2)}\right)^{2}-\left(a_{i^{\prime \prime}}^{(2)}\right)^{2}\right)\left(\left(a_{k^{\prime \prime}}^{(2)}\right)^{2}-\left(a_{i^{\prime \prime}}^{(2)}\right)^{2}\right)}, \\
i^{\prime \prime}=1^{\prime \prime}, 2^{\prime \prime}, 3^{\prime \prime} ; \quad\left(i^{\prime \prime} \neq j^{\prime \prime} \neq k^{\prime \prime} \neq i^{\prime \prime}\right), \quad(36) \\
-\left(a_{1^{\prime \prime}}^{(2)}\right)^{2}<\xi^{\prime \prime}<-\left(a_{2^{\prime \prime}}^{(2)}\right)^{2}<\eta^{\prime \prime}<-\left(a_{3^{\prime \prime}}^{(2)}\right)^{2}<\xi^{\prime \prime}<+\infty .
\end{gathered}
$$

$\mathbf{N}_{0}^{\prime \prime}\left(\xi^{\prime \prime}\right)-$ тензорная функция координаты $\xi^{\prime \prime}$, преобразованная к системе координат $x^{1^{\prime}} x^{2^{\prime}} x^{3^{\prime}}$ по формуле

$$
\mathbf{N}_{0}^{\prime \prime}\left(\xi^{\prime \prime}\right)=\left(\mathbf{T}_{1}^{-1}\right)^{T} \mathbf{N}^{\prime \prime}\left(\xi^{\prime \prime}\right) \mathbf{T}_{1}^{\prime-1},
$$

где $\mathbf{N}^{\prime \prime}\left(\xi^{\prime \prime}\right)$ - эта же функция в системе $x^{1^{\prime \prime}} x^{2^{\prime \prime}} x^{3^{\prime \prime}}$ :

$$
\begin{gathered}
\mathbf{N}^{\prime \prime}\left(\xi^{\prime \prime}\right)=\left\|\begin{array}{ccc}
N_{1^{\prime \prime}}^{\prime \prime}\left(\xi^{\prime \prime}\right) & 0 & 0 \\
0 & N_{2^{\prime \prime}}^{\prime \prime}\left(\xi^{\prime \prime}\right) & 0 \\
0 & 0 & N_{3^{\prime \prime}}^{\prime \prime}\left(\xi^{\prime \prime}\right)
\end{array}\right\|, \quad(37) \\
N_{i^{\prime \prime}}^{\prime \prime}\left(\xi^{\prime \prime}\right)=\frac{\bar{a}^{\prime \prime(2)}}{2} \int_{\xi^{\prime \prime}}^{+\infty} \frac{d u}{\left[u+\left(a_{i^{\prime \prime}}^{(2)}\right)^{2}\right] R_{u}^{\prime \prime(2)}}, \quad i^{\prime \prime}=1^{\prime \prime}, 2^{\prime \prime}, 3^{\prime \prime}, \\
R_{u}^{\prime \prime(k)}=\left[\left(u+\left(a_{1^{\prime \prime}}^{(k)}\right)^{2}\right)\left(u+\left(a_{2^{\prime \prime}}^{(k)}\right)^{2}\right)\left(u+\left(a_{3^{\prime \prime}}^{(k)}\right)^{2}\right)\right]^{1 / 2}, \\
k=1,2, \\
\bar{a}^{\prime \prime(k)}=a_{1^{\prime \prime}}^{(k)} a_{2^{\prime \prime}}^{(k)} a_{3^{\prime \prime}}^{(k)}, \quad k=1,2 .
\end{gathered}
$$

В выражениях (30) $\mathbf{L}^{\prime(1)}-$ тензор геометрических факторов эллипсоида $V^{\prime}$ с поверхностью $S_{1}^{\prime}$ :

$$
\mathbf{L}^{\prime(1)}=\mathbf{N}^{\prime}(0) ;
$$

$\mathbf{L}_{0}^{\prime \prime(i)}, i=1,2-$ преобразованные к системе $x^{1^{\prime}} x^{2^{\prime}} x^{3^{\prime}}$ тензоры геометрических факторов эллипсоидов, ограниченных снаружи поверхностями $S_{i}^{\prime \prime}, i=1,2$ соответственно:

$$
\mathbf{L}_{0}^{\prime \prime(i)}=\left(\mathbf{T}_{1}^{\prime-1}\right)^{T} \mathbf{L}^{\prime \prime(i)} \mathbf{T}_{1}^{\prime-1}, \quad i=1,2,
$$

где $\mathbf{L}^{\prime \prime(i)}, i=1,2-$ эти же тензоры в системе $x^{1^{\prime \prime}} x^{2^{\prime \prime}} x^{3^{\prime \prime}}$; их главные компоненты

$$
\begin{gathered}
L_{i^{\prime \prime}}^{\prime \prime(k)}=\frac{\bar{a}^{\prime \prime(k)}}{2} \int_{0}^{+\infty} \frac{d u}{\left[u+\left(a_{i^{\prime \prime}}^{(k)}\right)^{2}\right] R_{u}^{\prime \prime(k)}}, \\
i^{\prime \prime}=1^{\prime \prime}, 2^{\prime \prime}, 3^{\prime \prime} ; \quad k=1,2 ;
\end{gathered}
$$

$v^{\prime}$ - отношение объемов внутреннего $V_{2}^{\prime}$ и внешнего эллипсоидов $V^{\prime}$, которое в силу линейности преобразования (7) равно отношению объема ядра к объему всего включения:

$$
v^{\prime}=\bar{a}^{(2)} / \bar{a}^{\prime(1)}=\bar{a}^{(2)} / \bar{a}^{(1)} .
$$

Выражения для напряженности электрического поля в матрице (вакууме) и в оболочке имеют вид [6]

$$
\begin{aligned}
\mathbf{E}_{m}^{\prime}= & \left(\mathbf{I}+3\left[-\left(\bar{a}^{\prime(1)}\right)^{-1} \mathbf{N}^{\prime}\left(\xi^{\prime}\right)+h_{1}^{\prime-2}\left(R_{\xi^{\prime}}^{\prime(1)}\right)^{-1}\right.\right. \\
& \left.\left.\times\left(\mathbf{r}_{\xi^{\prime}}^{\prime} \otimes \mathbf{r}_{\xi^{\prime}}^{\prime}\right)\right] \boldsymbol{\alpha}^{\prime}\right) \mathbf{E}_{0}^{\prime}, \\
\mathbf{E}_{1}^{\prime}= & \left(-\boldsymbol{\beta}^{\prime(1)}+\left[-\mathbf{N}_{0}^{\prime \prime}\left(\xi^{\prime \prime}\right)+\bar{a}^{\prime \prime(2)} h_{1}^{\prime \prime-2}\left(R_{\xi^{\prime \prime}}^{\prime \prime(2)}\right)^{-1}\right.\right. \\
\times & \left.\left.\left(\mathbf{r}_{\xi^{\prime \prime}}^{\prime \prime} \otimes \mathbf{r}_{\xi^{\prime \prime}}^{\prime \prime}\right)_{0}\right] \boldsymbol{\alpha}^{\prime(1)}\right) \mathbf{E}_{0}^{\prime},
\end{aligned}
$$

где

$$
\mathbf{r}_{\xi^{\prime}}^{\prime} \equiv \frac{\partial \mathbf{r}^{\prime}}{\partial \xi^{\prime}}=\frac{1}{2}\left(\frac{x^{1^{\prime}}}{\xi^{\prime}+\left(a_{1^{\prime}}^{(1)}\right)^{2}} \frac{x^{2^{\prime}}}{\xi^{\prime}+\left(a_{2^{\prime}}^{(1)}\right)^{2}} \frac{x^{3^{\prime}}}{\xi^{\prime}+\left(a_{3^{\prime}}^{(1)}\right)^{2}}\right)^{T},
$$


$\mathbf{r}_{\xi^{\prime \prime}}^{\prime \prime} \equiv \frac{\partial \mathbf{r}^{\prime \prime}}{\partial \xi^{\prime \prime}}=\frac{1}{2}\left(\frac{x^{1^{\prime \prime}}}{\xi^{\prime \prime}+\left(a_{1^{\prime \prime}}^{(2)}\right)^{2}} \frac{x^{2^{\prime \prime}}}{\xi^{\prime \prime}+\left(a_{2^{\prime \prime}}^{(2)}\right)^{2}} \frac{x^{3^{\prime \prime}}}{\xi^{\prime \prime}+\left(a_{3^{\prime \prime}}^{(2)}\right)^{2}}\right)^{T}$,

$$
\left(\mathbf{r}_{\xi^{\prime \prime}}^{\prime \prime} \otimes \mathbf{r}_{\xi^{\prime \prime}}^{\prime \prime}\right)_{0}=\left(\mathbf{T}_{1}^{\prime-1}\right)^{T}\left(\mathbf{r}_{\xi^{\prime \prime}}^{\prime \prime} \otimes \mathbf{r}_{\xi^{\prime \prime}}^{\prime \prime}\right) \mathbf{T}_{1}^{\prime-1}
$$

- тензор, преобразованный к системе $x^{1^{\prime}} x^{2^{\prime}} x^{3^{\prime}}$, $\left(\mathbf{r}_{\xi^{\prime \prime}}^{\prime \prime} \otimes \mathbf{r}_{\xi^{\prime \prime}}^{\prime \prime}\right)$ - этот же тензор в системе $x^{1^{\prime \prime}} x^{2^{\prime \prime}} x^{3^{\prime \prime}}$;

$$
\begin{aligned}
h_{1}^{\prime} & =\left(2 R_{\xi^{\prime}}^{(1)}\right)^{-1} \sqrt{\left(\xi^{\prime}-\eta^{\prime}\right)\left(\xi^{\prime}-\xi^{\prime}\right)}, \\
h_{1}^{\prime \prime} & =\left(2 R_{\xi^{\prime \prime}}^{\prime \prime(2)}\right)^{-1} \sqrt{\left(\xi^{\prime \prime}-\eta^{\prime \prime}\right)\left(\xi^{\prime \prime}-\xi^{\prime \prime}\right)}
\end{aligned}
$$

- первые из коэффициентов Ламе соответствующих эллипсоидальных систем координат. Напряженность электрического поля в ядре включения имеет вид (29).

\section{Получение решения исходной задачи с помощью преобразования решения задачи для включения с оболочкой в вакууме}

Чтобы получить решение исходной задачи (1)-(6), нужно выражения (28)-(30), (42) преобразовать к исходной системе координат $x^{1} x^{2} x^{3}$. Тензорные величины, определяемые выражениями (30), преобразуются при переходе к системе $x^{1} x^{2} x^{3}$ по формулам (обозначаются без штрихов):

$$
\begin{gathered}
\lambda_{20}=\left(\mathbf{T}_{m}^{-1}\right)^{T} \lambda_{20}^{\prime} \mathbf{T}_{m}^{T}, \quad \boldsymbol{\alpha}=\mathbf{T}_{m} \boldsymbol{\alpha}^{\prime} \mathbf{T}_{m}^{T}, \\
\boldsymbol{\alpha}^{(1)}=\mathbf{T}_{m} \boldsymbol{\alpha}^{\prime(1)} \mathbf{T}_{m}^{T}, \quad \boldsymbol{\beta}^{(1)}=\left(\mathbf{T}_{m}^{-1}\right)^{T} \boldsymbol{\beta}^{\prime(1)} \mathbf{T}_{m}^{T},
\end{gathered}
$$

так как $\lambda_{20}$ и $\boldsymbol{\beta}^{(1)}$ - ковариантно-контравариантные, а $\boldsymbol{\alpha}$ и $\boldsymbol{\alpha}^{(1)}$ - дважды контравариантные. Элементарными преобразованиями получим для них выражения

$$
\begin{aligned}
\lambda_{20}= & {\left[\left(\mathbf{I}+\mathbf{L}_{0}^{\prime(1)}\left(\boldsymbol{\varepsilon}_{1}-\boldsymbol{\varepsilon}_{m}\right)\right)\left(\mathbf{I}+\left(\mathbf{L}_{00}^{\prime \prime(2)}-v^{\prime} \mathbf{L}_{00}^{\prime \prime(1)}\right)\left(\boldsymbol{\varepsilon}_{2}-\boldsymbol{\varepsilon}_{1}\right)\right)\right.} \\
& \left.+v^{\prime} \mathbf{L}_{0}^{\prime(1)}\left(\boldsymbol{\varepsilon}_{2}-\boldsymbol{\varepsilon}_{1}\right)\right]^{-1}, \\
\boldsymbol{\alpha}= & 3^{-1} \bar{a}^{\prime(1)}\left[\left(\boldsymbol{\varepsilon}_{1}-\boldsymbol{\varepsilon}_{m}\right)\right)\left(\mathbf{I}+\left(\mathbf{L}_{00}^{\prime \prime(2)}-v^{\prime} \mathbf{L}_{00}^{\prime \prime(1)}\right)\left(\boldsymbol{\varepsilon}_{2}-\boldsymbol{\varepsilon}_{1}\right)\right) \\
& \left.+v^{\prime}\left(\boldsymbol{\varepsilon}_{2}-\boldsymbol{\varepsilon}_{1}\right)\right] \boldsymbol{\lambda}_{20}, \\
\boldsymbol{\alpha}^{(1)}= & \left(\boldsymbol{\varepsilon}_{2}-\boldsymbol{\varepsilon}_{1}\right) \boldsymbol{\lambda}_{20}, \quad \boldsymbol{\beta}^{(1)}=-\left(\mathbf{I}+\mathbf{L}_{00}^{\prime \prime(2)}\left(\boldsymbol{\varepsilon}_{2}-\boldsymbol{\varepsilon}_{1}\right)\right) \boldsymbol{\lambda}_{20},
\end{aligned}
$$

где

$$
\mathbf{L}_{0}^{\prime(1)}=\left(\mathbf{T}_{m}^{-1}\right)^{T} \mathbf{L}^{\prime(1)} \mathbf{T}_{m}^{-1}
$$

- тензор геометрических факторов эллипсоида с поверхностью $S_{1}^{\prime}$ в системе координат $x^{1} x^{2} x^{3}$ (он дважды ковариантный);

$$
\mathbf{L}_{00}^{\prime \prime(i)}=\left(\mathbf{T}_{m}^{-1}\right)^{T} \mathbf{L}_{0}^{\prime \prime(i)} \mathbf{T}_{m}^{-1}, \quad i=1,2
$$

- тензоры геометрических факторов эллипсоидов с поверхностями $S_{i}^{\prime \prime}, i=1,2$ соответственно в системе $x^{1} x^{2} x^{3}$
Преобразуя выражения (28), (29), (42) к системе координат $x^{1} x^{2} x^{3}$, в итоге получим для потенциала и напряженности электрического поля в матрице, оболочке и ядре включения следующие выражения:

$$
\begin{aligned}
& \varphi_{m}(\mathbf{r})=\left(\left(-\mathbf{I}+3\left(\bar{a}^{\prime(1)}\right)^{-1} \mathbf{N}_{0}^{\prime}\left(\xi^{\prime}\right) \boldsymbol{\alpha}\right) \mathbf{E}_{0}, \mathbf{r}\right), \mathbf{r} \in V_{m}, \xi^{\prime} \geq 0, \\
& \varphi_{1}(\mathbf{r})=\left(\left(\boldsymbol{\beta}^{(1)}+\mathbf{N}_{00}^{\prime \prime}\left(\xi^{\prime \prime}\right) \boldsymbol{\alpha}^{(1)}\right) \mathbf{E}_{0}, \mathbf{r}\right), \mathbf{r} \in V_{1}, 0 \leq \xi^{\prime \prime} \leq t^{\prime \prime}, \\
& \varphi_{2}(\mathbf{r})=-\left(\boldsymbol{\lambda}_{20} \mathbf{E}_{0}, \mathbf{r}\right), \\
& \mathbf{E}_{m}=\left(\mathbf{I}+3\left[-\left(\bar{a}^{\prime(1)}\right)^{-1} \mathbf{N}_{0}^{\prime}\left(\xi^{\prime}\right)+h_{1}^{\prime-2}\left(R_{\xi^{\prime}}^{(1)}\right)^{-1}\right.\right. \\
&\left.\left.\times\left(\mathbf{r}_{\xi^{\prime}}^{\prime} \otimes \mathbf{r}_{\xi^{\prime}}^{\prime}\right)_{0}\right] \boldsymbol{\alpha}\right) \mathbf{E}_{0}, \\
& \mathbf{E}_{1}=\left(-\boldsymbol{\beta}^{(1)}+\left[-\mathbf{N}_{00}^{\prime \prime}\left(\xi^{\prime \prime}\right)+\bar{a}^{\prime \prime(2)} h_{1}^{\prime \prime-2}\left(R_{\xi^{\prime \prime}}^{\prime \prime(2)}\right)^{-1}\right.\right. \\
&\left.\left.\times\left(\mathbf{r}_{\xi^{\prime \prime}}^{\prime \prime} \otimes \mathbf{r}_{\xi^{\prime \prime}}^{\prime \prime}\right)_{00}\right] \boldsymbol{\alpha}^{(1)}\right) \mathbf{E}_{0}, \\
& \mathbf{E}_{2}=\lambda_{20} \mathbf{E}_{0},
\end{aligned}
$$

где

$$
\begin{aligned}
\mathbf{N}_{0}^{\prime}\left(\xi^{\prime}\right)= & \left(\mathbf{T}_{m}^{-1}\right)^{T} \mathbf{N}^{\prime}\left(\xi^{\prime}\right) \mathbf{T}_{m}^{-1}, \mathbf{N}_{00}^{\prime \prime}\left(\xi^{\prime \prime}\right)=\left(\mathbf{T}_{m}^{-1}\right)^{T} \mathbf{N}_{0}^{\prime \prime}\left(\xi^{\prime \prime}\right) \mathbf{T}_{m}^{-1}, \\
& \left(\mathbf{r}_{\xi^{\prime}}^{\prime} \otimes \mathbf{r}_{\xi^{\prime}}^{\prime}\right)_{0}=\left(\mathbf{T}_{m}^{-1}\right)^{T}\left(\mathbf{r}_{\xi^{\prime}}^{\prime} \otimes \mathbf{r}_{\xi^{\prime}}^{\prime}\right) \mathbf{T}_{m}^{-1}, \\
& \left(\mathbf{r}_{\xi^{\prime \prime}}^{\prime \prime} \otimes \mathbf{r}_{\xi^{\prime \prime}}^{\prime \prime}\right)_{00}=\left(\mathbf{T}_{m}^{-1}\right)^{T}\left(\mathbf{r}_{\xi^{\prime \prime}}^{\prime \prime} \otimes \mathbf{r}_{\xi^{\prime \prime}}^{\prime \prime}\right)_{0} \mathbf{T}_{m}^{-1}
\end{aligned}
$$

- соответствующие тензорные величины в системе $x^{1} x^{2} x^{3}$.

\section{Дипольный момент и тензор поляризуемости включения с оболочкой в анизотропной среде}

Потенциал точечного заряда $q$ в анизотропной среде, расположенного в начале координат, имеет выражение $[10]$

$$
\varphi_{q}=\left(\operatorname{det} \varepsilon_{m}\right)^{-1 / 2}\left(\mathbf{r}^{T} \varepsilon_{m}^{-1} \mathbf{r}\right)^{-1 / 2} q,
$$

где $\boldsymbol{\varepsilon}_{m}-$ тензор диэлектрической проницаемости среды. Потенциал такого же по абсолютной величине, но противоположного по знаку заряда $-q$, расположенного в близкой к началу координат точке $-\mathrm{dr}$, равен

$$
\varphi_{-q}^{\prime}=-\left(\operatorname{det} \boldsymbol{\varepsilon}_{m}\right)^{-1 / 2}\left((\mathbf{r}+\mathrm{d} \mathbf{r})^{T} \boldsymbol{\varepsilon}_{m}^{-1}(\mathbf{r}+\mathrm{d} \mathbf{r})\right)^{-1 / 2} q .
$$

Складывая (47) и (48) и линеаризуя по dr, получим потенциал точечного диполя с моментом $\mathbf{p}=q \mathrm{~d} \mathbf{r}$ в анизотропной среде

$$
\varphi_{\mathbf{p}}=\left(\operatorname{det} \boldsymbol{\varepsilon}_{m}\right)^{-1 / 2}\left(\mathbf{r}^{T} \boldsymbol{\varepsilon}_{m}^{-1} \mathbf{r}\right)^{-3 / 2}\left(\boldsymbol{\varepsilon}_{m}^{-1} \mathbf{p}, \mathbf{r}\right) .
$$

Для нахождения дипольного момента включения с оболочкой рассмотрим первое из выражений (44) для потенциала поля $\varphi_{m}$ в матрице в асимптотике при $r \rightarrow \infty$. Так как $\xi^{\prime} \approx r^{\prime 2} \equiv \mathbf{r}^{\prime 2}, \xi^{\prime} \rightarrow+\infty$, то

$$
\mathbf{N}^{\prime}\left(\xi^{\prime}\right) \approx 3^{-1} \bar{a}^{\prime(1)} \xi^{\prime-3 / 2} \mathbf{I} \approx 3^{-1} \bar{a}^{\prime(1)} r^{\prime-3} \mathbf{I}, \quad \xi^{\prime} \rightarrow+\infty,
$$




$$
\begin{gathered}
\mathbf{N}_{0}^{\prime}\left(\xi^{\prime}\right) \approx 3^{-1} \bar{a}^{\prime(1)} r^{\prime-3}\left(\mathbf{T}_{m}^{-1}\right)^{T} \mathbf{I} \mathbf{T}_{m}^{-1}=3^{-1} \bar{a}^{\prime(1)} r^{\prime-3} \boldsymbol{\varepsilon}_{m}^{-1}, \\
\xi^{\prime} \rightarrow+\infty .
\end{gathered}
$$

Поскольку $r^{\prime 2}=\left(\mathbf{r}^{T} \varepsilon_{m}^{-1} \mathbf{r}\right)$, то

$$
\mathbf{N}_{0}^{\prime}\left(\xi^{\prime}\right) \approx 3^{-1} \bar{a}^{\prime(1)}\left(\mathbf{r}^{T} \boldsymbol{\varepsilon}_{m}^{-1} \mathbf{r}\right)^{-3 / 2} \boldsymbol{\varepsilon}_{m}^{-1}, \quad \xi^{\prime}, r \rightarrow+\infty,
$$

и для $\varphi_{m}$ получим

$$
\varphi_{m} \approx\left(\left(-\mathbf{I}+\left(\mathbf{r}^{T} \boldsymbol{\varepsilon}_{m}^{-1} \mathbf{r}\right)^{-3 / 2} \boldsymbol{\varepsilon}_{m}^{-1} \boldsymbol{\alpha}\right) \mathbf{E}_{0}, \mathbf{r}\right), \quad r \rightarrow+\infty,
$$

причем потенциал возмущенного поля

$$
\varphi_{p}=\varphi_{m}-\varphi_{0} \approx\left(\left(\mathbf{r}^{T} \boldsymbol{\varepsilon}_{m}^{-1} \mathbf{r}\right)^{-3 / 2} \boldsymbol{\varepsilon}_{m}^{-1} \boldsymbol{\alpha} \mathbf{E}_{0}, \mathbf{r}\right), \quad r \rightarrow+\infty .
$$

Сопоставляя это выражение с потенциалом точечного диполя (49), найдем дипольный момент включения с оболочкой

$$
\mathbf{p}=\left(\operatorname{det} \boldsymbol{\varepsilon}_{m}\right)^{1 / 2} \boldsymbol{\alpha} \mathbf{E}_{0},
$$

откуда видно, что тензор поляризуемости включения

$$
\tilde{\boldsymbol{\alpha}}=\left(\operatorname{det} \boldsymbol{\varepsilon}_{m}\right)^{1 / 2} \boldsymbol{\alpha},
$$

где $\boldsymbol{\alpha}$ определяется формулой (43). Поскольку

$$
\left(\operatorname{det} \varepsilon_{m}\right)^{1 / 2} \bar{a}^{(1)}=\bar{a}^{(1)},
$$

перепишем выражение для тензора поляризуемости включения в виде

$$
\begin{aligned}
\tilde{\boldsymbol{\alpha}}= & 3^{-1} \bar{a}^{(1)}\left[\left(\boldsymbol{\varepsilon}_{1}-\boldsymbol{\varepsilon}_{m}\right)\left(\mathbf{I}+\left(\mathbf{L}_{00}^{\prime \prime(2)}-v^{\prime} \mathbf{L}_{00}^{\prime \prime(1)}\right)\left(\boldsymbol{\varepsilon}_{2}-\boldsymbol{\varepsilon}_{1}\right)\right)\right. \\
& \left.+v^{\prime}\left(\boldsymbol{\varepsilon}_{2}-\boldsymbol{\varepsilon}_{1}\right)\right] \lambda_{20} .
\end{aligned}
$$

\section{Включение с изотропной оболочкой в анизотропной среде}

Рассмотрим частный, но важный для приложений случай, когда оболочка включения имеет изотропные материальные характеристики, т. е. тензор диэлектрической проницаемости оболочки имеет вид

$$
\varepsilon_{1}=\varepsilon_{1} \mathbf{I}
$$

В этом случае, очевидно, преобразование (35), устраняющее анизотропию оболочки после преобразования (7), т. е. сводящее $\varepsilon_{1}^{\prime}$ к единичному тензору $\mathbf{I}$, есть

$$
\mathbf{T}_{1}^{\prime}=\mathbf{T}_{m}^{-1} \sqrt{\varepsilon_{1}} \mathbf{I},
$$

При этом координатные системы $x^{1^{\prime \prime}} x^{2^{\prime \prime}} x^{3^{\prime \prime}}$ и $x^{1} x^{2} x^{3}$ связаны между собой соотношениями

$$
x^{i}=\sqrt{\varepsilon_{1}} x^{i^{\prime \prime}}, \quad i=1,2,3 ; i^{\prime \prime}=1^{\prime \prime}, 2^{\prime \prime}, 3^{\prime \prime},
$$

или в векторном виде

$$
\mathbf{r}=\mathbf{T}_{1} \mathbf{r}^{\prime \prime}, \quad \mathbf{T}_{1}=\sqrt{\varepsilon_{1}} \mathbf{I} .
$$

Софокусность поверхностей-эллипсоидов при изотропном растяжении сохраняется, поэтому софокусными считаются исходные поверхности-эллипсоиды $S_{1}, S_{2}$, т. е.

$$
\left(a_{k}^{(1)}\right)^{2}=\left(a_{k}^{(2)}\right)^{2}+t, \quad k=1,2,3,
$$

где $t>0-$,шаг софокусности“. Полуоси поверхностейэллипсоидов $S_{i}$ и $S_{i}^{\prime \prime}, i=1,2$ связаны между собой, согласно (53):

$$
a_{k^{\prime \prime}}^{(i)}=\left(\varepsilon_{1}\right)^{-1 / 2} a_{k}^{(i)}, k=1,2,3 ; k^{\prime \prime}=1^{\prime \prime}, 2^{\prime \prime}, 3^{\prime \prime} ; i=1,2,
$$

при этом для „шага софокусности“ поверхностей $S_{1}, S_{2}$ имеем

$$
t=\varepsilon_{1} t^{\prime \prime}
$$

Введем эллипсоидальные координаты $\xi, \eta, \xi$, связанные с координатами $x^{1}, x^{2}, x^{3}$ по формулам:

$$
\begin{gathered}
\left(x^{i}\right)^{2}=\frac{\left(\xi+\left(a_{i}^{(2)}\right)^{2}\right)\left(\eta+\left(a_{i}^{(2)}\right)^{2}\right)\left(\xi+\left(a_{i}^{(2)}\right)^{2}\right)}{\left(\left(a_{j}^{(2)}\right)^{2}-\left(a_{i}^{(2)}\right)^{2}\right)\left(\left(a_{k}^{(2)}\right)^{2}-\left(a_{i}^{(2)}\right)^{2}\right)}, \\
i=1,2,3 ; \quad(i \neq j \neq k \neq i),
\end{gathered}
$$

где $-\left(a_{1}^{(2)}\right)^{2}<\xi<-\left(a_{2}^{(2)}\right)^{2}<\eta<-\left(a_{3}^{(2)}\right)^{2}<\xi<+\infty$. Значению координаты $\xi=0$ соответствует положение точек на внутренней поверхности $S_{2}$. Связь координат $\xi, \eta, \xi$ с координатами $\xi^{\prime \prime}, \eta^{\prime \prime}, \xi^{\prime \prime}$, введенными формулами (36), имеет вид

$$
\xi=\varepsilon_{1} \xi^{\prime \prime}, \quad \eta=\varepsilon_{1} \eta^{\prime \prime}, \quad \xi=\varepsilon_{1} \xi^{\prime \prime} .
$$

Введем тензорную функцию $\mathbf{N}(\xi)$ переменной $\xi$, имеющую в системе $x^{1} x^{2} x^{3}$ вид

$$
\mathbf{N}(\xi)=\left\|\begin{array}{ccc}
N_{1}(\xi) & 0 & 0 \\
0 & N_{2}(\xi) & 0 \\
0 & 0 & N_{3}(\xi)
\end{array}\right\|,
$$

$N_{i}(\xi)=\frac{\bar{a}^{(2)}}{2} \int_{\xi}^{+\infty} \frac{d u}{\left[u+\left(a_{i}^{(2)}\right)^{2}\right] R_{u}^{(2)}}, \quad i=1,2,3 ; 0 \leq \xi \leq t$,

где

$$
\begin{gathered}
R_{u}^{(k)}=\left[\left(u+\left(a_{1}^{(k)}\right)^{2}\right)\left(u+\left(a_{2}^{(k)}\right)^{2}\right)\left(u+\left(a_{3}^{(k)}\right)^{2}\right)\right]^{1 / 2}, \\
k=1,2 .
\end{gathered}
$$

С помощью элементарных преобразований нетрудно убедиться, что

$$
\mathbf{N}^{\prime \prime}\left(\xi^{\prime \prime}\right)=\mathbf{N}(\xi),
$$

где $\mathbf{N}^{\prime \prime}\left(\xi^{\prime \prime}\right)$ - тензорная функция, определяемая выражениями (37)-(40). Тогда для этой же тензорной функции в системе $x^{1} x^{2} x^{3}$ получим

$$
\mathbf{N}_{00}^{\prime \prime}\left(\xi^{\prime \prime}\right)=\left(\mathbf{T}_{1}^{-1}\right)^{T} \mathbf{N}^{\prime \prime}\left(\xi^{\prime \prime}\right) \mathbf{T}_{1}^{-1}=\varepsilon_{1}^{-1} \mathbf{N}(\xi) .
$$


Далее, так как для тензоров геометрических факторов эллипсоидов с поверхностями $S_{1}^{\prime \prime}, S_{2}^{\prime \prime}$ имеют место соотношения $\mathbf{L}_{00}^{\prime \prime(2)}=\mathbf{N}_{00}^{\prime \prime}(0), \mathbf{L}_{00}^{\prime \prime(1)}=v^{\prime-1} \mathbf{N}_{00}^{\prime \prime}\left(t^{\prime \prime}\right)$, то

$$
\mathbf{L}_{00}^{\prime \prime(k)}=\varepsilon_{1}^{-1} \mathbf{L}^{(k)}, \quad k=1,2,
$$

где $\mathbf{L}^{(1)}, \mathbf{L}^{(2)}$ - тензоры геометрических факторов эллипсоидов с поверхностями $S_{1}, S_{2}$ соответственно:

$$
\mathbf{L}^{(1)}=v^{\prime-1} \mathbf{N}(t), \quad \mathbf{L}^{(2)}=\mathbf{N}(0),
$$

их главные компоненты

$$
L_{i}^{(k)}=\frac{\bar{a}^{(k)}}{2} \int_{0}^{+\infty} \frac{d u}{\left[u+\left(a_{i}^{(k)}\right)^{2}\right] R_{u}^{(k)}}, \quad i=1,2,3 ; k=1,2 .
$$

Упростим выражение для тензора $\left(\mathbf{r}_{\xi^{\prime \prime}}^{\prime \prime} \otimes \mathbf{r}_{\xi^{\prime \prime}}^{\prime \prime}\right)_{00}$ в случае изотропной оболочки. С учетом (53) и $(55)$

$$
\mathbf{r}_{\xi^{\prime \prime}}^{\prime \prime}=\sqrt{\varepsilon_{1}} \mathbf{r}_{\xi},
$$

где

$$
\mathbf{r}_{\xi} \equiv \frac{\partial \mathbf{r}}{\partial \xi}=\frac{1}{2}\left(\frac{x^{1}}{\xi+\left(a_{1}^{(2)}\right)^{2}} \frac{x^{2}}{\xi+\left(a_{2}^{(2)}\right)^{2}} \frac{x^{3}}{\xi+\left(a_{3}^{(2)}\right)^{2}},\right)^{T},
$$

тогда

$$
\mathbf{r}_{\xi^{\prime \prime}}^{\prime \prime} \otimes \mathbf{r}_{\xi^{\prime \prime}}^{\prime \prime}=\varepsilon_{1}\left(\mathbf{r}_{\xi} \otimes \mathbf{r}_{\xi}\right)
$$

поэтому

$$
\left(\mathbf{r}_{\xi^{\prime \prime}}^{\prime \prime} \otimes \mathbf{r}_{\xi^{\prime \prime}}^{\prime \prime}\right)_{00}=\varepsilon_{1}^{-1}\left(\mathbf{r}_{\xi^{\prime \prime}}^{\prime \prime} \otimes \mathbf{r}_{\xi^{\prime \prime}}^{\prime \prime}\right)=\left(\mathbf{r}_{\xi} \otimes \mathbf{r}_{\xi}\right),
$$

Для коэффициента Ламе $h_{1}^{\prime \prime}$ и выражения $R_{\xi^{\prime \prime}}^{\prime \prime(2)}$ имеем

$$
h_{1}^{\prime \prime}=\sqrt{\varepsilon_{1}} h_{1}, \quad R_{\xi^{\prime \prime}}^{\prime \prime(2)}=\varepsilon_{1}^{-3 / 2} R_{\xi}^{(2)},
$$

где

$$
h_{1}=\left(2 R_{\xi}^{(2)}\right)^{-1} \sqrt{(\xi-\eta)(\xi-\zeta)} .
$$

С учетом соотношений (52), (59) выражения (43) для тензоров $\lambda_{20}, \boldsymbol{\alpha}, \boldsymbol{\alpha}^{(1)}, \boldsymbol{\beta}^{(1)}$ принимают вид

$$
\begin{aligned}
& \lambda_{20}= {\left[( \mathbf { I } + \mathbf { L } _ { 0 } ^ { \prime ( 1 ) } ( \varepsilon _ { 1 } \mathbf { I } - \boldsymbol { \varepsilon } _ { m } ) ) \left(\mathbf{I}+\varepsilon_{1}^{-1}\left(\mathbf{L}^{(2)}-v^{\prime} \mathbf{L}^{(1)}\right)\right.\right.} \\
&\left.\left.\times\left(\boldsymbol{\varepsilon}_{2}-\varepsilon_{1} \mathbf{I}\right)\right)+v^{\prime} \mathbf{L}_{0}^{\prime(1)}\left(\boldsymbol{\varepsilon}_{2}-\varepsilon_{1} \mathbf{I}\right)\right]^{-1}, \\
& \boldsymbol{\alpha}= 3^{-1} \bar{a}^{\prime(1)}\left[\left(\varepsilon_{1} \mathbf{I}-\boldsymbol{\varepsilon}_{m}\right)\right)\left(\mathbf{I}+\varepsilon_{1}^{-1}\left(\mathbf{L}^{(2)}-v^{\prime} \mathbf{L}^{(1)}\right)\right. \\
&\left.\left.\times\left(\boldsymbol{\varepsilon}_{2}-\varepsilon_{1} \mathbf{I}\right)\right)+v^{\prime}\left(\boldsymbol{\varepsilon}_{2}-\varepsilon_{1} \mathbf{I}\right)\right] \lambda_{20}, \\
& \boldsymbol{\alpha}^{(1)}=\left(\boldsymbol{\varepsilon}_{2}-\varepsilon_{1} \mathbf{I}\right) \boldsymbol{\lambda}_{20}, \boldsymbol{\beta}^{(1)}=-\left(\mathbf{I}+\varepsilon_{1}^{-1} \mathbf{L}^{(2)}\left(\boldsymbol{\varepsilon}_{2}-\varepsilon_{1} \mathbf{I}\right)\right) \boldsymbol{\lambda}_{20} .
\end{aligned}
$$

Выражения для потенциала и напряженности электрического поля в матрице и ядре остаются такими же, как и в (44), (45), а выражения для них в оболочке с учетом (58), (61), (62) немного упрощаются. Выпишем для удобства все указанные выражения

$$
\begin{gathered}
\varphi_{m}(\mathbf{r})=\left(\left(-\mathbf{I}+3\left(\bar{a}^{\prime(1)}\right)^{-1} \mathbf{N}_{0}^{\prime}\left(\xi^{\prime}\right) \boldsymbol{\alpha}\right) \mathbf{E}_{0}, \mathbf{r}\right), \mathbf{r} \in V_{m}, \xi^{\prime} \geq 0, \\
\varphi_{1}(\mathbf{r})=\left(\left(\boldsymbol{\beta}^{(1)}+\varepsilon_{1}^{-1} \mathbf{N}(\xi) \boldsymbol{\alpha}^{(1)}\right) \mathbf{E}_{0}, \mathbf{r}\right), \mathbf{r} \in V_{1}, \quad 0 \leq \xi \leq t, \\
\varphi_{2}(\mathbf{r})=-\left(\boldsymbol{\lambda}_{20} \mathbf{E}_{0}, \mathbf{r}\right), \\
\mathbf{E}_{m}=\left(\mathbf{I}+3\left[-\left(\bar{a}^{\prime(1)}\right)^{-1} \mathbf{N}_{0}^{\prime}\left(\xi^{\prime}\right)+h_{1}^{\prime-2}\left(R_{\xi^{\prime}}^{\prime(1)}\right)^{-1}\right.\right. \\
\left.\left.\quad \times\left(\mathbf{r}_{\xi^{\prime}}^{\prime} \otimes \mathbf{r}_{\xi^{\prime}}^{\prime}\right)_{0}\right] \boldsymbol{\alpha}\right) \mathbf{E}_{0}, \\
\mathbf{E}_{1}=\left(-\boldsymbol{\beta}^{(1)}+\varepsilon_{1}^{-1}\left[-\mathbf{N}(\xi)+\bar{a}^{(2)} h_{1}^{-2}\left(R_{\xi}^{(2)}\right)^{-1}\right.\right. \\
\left.\left.\quad \times\left(\mathbf{r}_{\xi} \otimes \mathbf{r}_{\xi}\right)\right] \boldsymbol{\alpha}^{(1)}\right) \mathbf{E}_{0}, \\
\mathbf{E}_{2}=\lambda_{20} \mathbf{E}_{0},
\end{gathered}
$$

где $\mathbf{N}(\xi), R_{\xi}^{(2)},\left(\mathbf{r}_{\xi} \otimes \mathbf{r}_{\xi}\right), h_{1}$ определяются выражениями (56)-(57), (60), (61), (63) соответственно.

\section{Примеры}

1. Рассмотрим случай анизотропного шара с изотропной сферической оболочкой в анизотропной матрице. Пусть $a^{(1)}, a^{(2)}$ - радиусы внешней и внутренней сфер $S_{1}, S_{2}$. Направим оси системы координат $x^{1} x^{2} x^{3}$ вдоль главных осей тензора $\varepsilon_{m}$, тогда матрица преобразования (7) будет иметь в системе $x^{1} x^{2} x^{3}$ вид

$$
\mathbf{T}_{m}=\left(\begin{array}{ccc}
\left(\varepsilon_{1}^{(m)}\right)^{1 / 2} & 0 & 0 \\
0 & \left(\varepsilon_{2}^{(m)}\right)^{1 / 2} & 0 \\
0 & 0 & \left(\varepsilon_{3}^{(m)}\right)^{1 / 2}
\end{array}\right),
$$

где $\varepsilon_{k}^{(m)}, k=1,2,3$ - главные компоненты тензора $\varepsilon_{m}$. Сферы $S_{1}, S_{2}$ при преобразовании (7) переходят в поверхности-эллипсоиды $S_{1}^{\prime}, S_{2}^{\prime}$ с полуосями

$$
a_{k^{\prime}}^{(i)}=\left(\varepsilon_{k}^{(m)}\right)^{1 / 2} a^{(i)}, k=1,2,3 ; k^{\prime}=1^{\prime}, 2^{\prime}, 3^{\prime} ; i=1,2 .
$$

Для главных компонент тензорной функции $\mathbf{N}^{\prime}\left(\xi^{\prime}\right)$ в соответствии с (67) получаем

$$
\begin{gathered}
N_{k^{\prime}}^{\prime}\left(\xi^{\prime}\right)=\frac{\left(a^{(1)}\right)^{3}}{2\left(\varepsilon_{1}^{(m)} \varepsilon_{2}^{(m)} \varepsilon_{3}^{(m)}\right)^{1 / 2}} \int_{\xi^{\prime}}^{+\infty} \frac{d u}{\left[u+\left(\varepsilon_{k}^{(m)}\right)^{-1}\left(a^{(1)}\right)^{2}\right] R_{u}^{\prime(1)}}, \\
k^{\prime}=1^{\prime}, 2^{\prime}, 3^{\prime}
\end{gathered}
$$

где

$$
R_{u}^{\prime(1)}=\left[\prod_{k=1}^{3}\left(u+\left(\varepsilon_{k}^{(m)}\right)^{-1}\left(a^{(1)}\right)^{2}\right)\right]^{1 / 2},
$$

для главных компонент $\mathbf{N}_{0}^{\prime}\left(\xi^{\prime}\right)$ имеем

$$
N_{0, k^{\prime}}^{\prime}\left(\xi^{\prime}\right)=\left(\varepsilon_{k}^{(m)}\right)^{-1} N_{k^{\prime}}^{\prime}\left(\xi^{\prime}\right), k=1,2,3 ; k^{\prime}=1^{\prime}, 2^{\prime}, 3^{\prime} .
$$


В соответствии с (41) для главных компонент тензора $\mathbf{L}_{0}^{\prime(1)}$ имеем выражения

$$
L_{0, k^{\prime}}^{\prime(1)}=\left(\varepsilon_{k}^{(m)}\right)^{-1} N_{k^{\prime}}^{\prime}(0), k=1,2,3 ; k^{\prime}=1^{\prime}, 2^{\prime}, 3^{\prime} .
$$

Несложно проверить, что в случае вырождения поверхности-эллипсоида $S_{2}$ в сферу для координат $\xi, \eta, \xi$, определяемых формулами (54), получаются предельные выражения

$$
\xi \rightarrow r^{2}-\left(a^{(2)}\right)^{2}, \quad \eta, \xi \rightarrow-\left(a^{(2)}\right)^{2},
$$

поэтому непосредственным вычислением по формулам $(57),(63)$ для $\mathbf{N}(\xi), R_{\xi}^{(2)}, h_{1}$ получаем

$$
\mathbf{N}(\xi)=3^{-1}\left(a^{(2)} / r\right)^{3} \mathbf{I}, \quad R_{\xi}^{(2)}=r^{3}, \quad h_{1}=1 /(2 r) .
$$

Имеем также в данном случае

$$
\mathbf{r}_{\xi} \otimes \mathbf{r}_{\xi}=4^{-1} r^{-4} \mathbf{r} \otimes \mathbf{r}, \quad \mathbf{L}^{(1)}=\mathbf{L}^{(2)}=3^{-1} \mathbf{I} .
$$

Выражения (64) для тензоров $\lambda_{20}, \boldsymbol{\alpha}, \boldsymbol{\alpha}^{(1)}, \boldsymbol{\beta}^{(1)}$ принимают вид

$$
\begin{gathered}
\boldsymbol{\lambda}_{20}=\left[\left(\mathbf{I}+\mathbf{L}_{0}^{\prime(1)} \varepsilon_{1} \mathbf{I}-\boldsymbol{\varepsilon}_{m}\right)\right)\left(\mathbf{I}+3^{-1} \varepsilon_{1}^{-1}\left(1-v^{\prime}\right)\right. \\
\left.\left.\times\left(\boldsymbol{\varepsilon}_{2}-\varepsilon_{1} \mathbf{I}\right)\right)+v^{\prime} \mathbf{L}_{0}^{\prime(1)}\left(\boldsymbol{\varepsilon}_{2}-\varepsilon_{1} \mathbf{I}\right)\right]^{-1}, \\
\boldsymbol{\alpha}=3^{-1}\left(\varepsilon_{1}^{(m)} \varepsilon_{2}^{(m)} \varepsilon_{3}^{(m)}\right)^{-1 / 2}\left(a^{(1)}\right)^{3}\left[( \varepsilon _ { 1 } \mathbf { I } - \boldsymbol { \varepsilon } _ { m } ) \left(\mathbf{I}+3^{-1} \varepsilon_{1}^{-1}\right.\right. \\
\left.\left.\times\left(1-v^{\prime}\right)\left(\boldsymbol{\varepsilon}_{2}-\varepsilon_{1} \mathbf{I}\right)\right)+v^{\prime}\left(\boldsymbol{\varepsilon}_{2}-\varepsilon_{1} \mathbf{I}\right)\right] \boldsymbol{\lambda}_{20}, \\
\boldsymbol{\alpha}^{(1)}=\left(\boldsymbol{\varepsilon}_{2}-\varepsilon_{1} \mathbf{I}\right) \boldsymbol{\lambda}_{20}, \boldsymbol{\beta}^{(1)}=-\left(\mathbf{I}+3^{-1} \varepsilon_{1}^{-1}\left(\boldsymbol{\varepsilon}_{2}-\varepsilon_{1} \mathbf{I}\right)\right) \boldsymbol{\lambda}_{20} .
\end{gathered}
$$

Таким образом, потенциал и напряженность электрического поля в сферической оболочке задаются следующими выражениями:

$$
\begin{gathered}
\varphi_{1}(\mathbf{r})=\left(\left(\boldsymbol{\beta}^{(1)}+3^{-1} \varepsilon_{1}^{-1}\left(a^{(2)} / r\right)^{3} \boldsymbol{\alpha}^{(1)}\right) \mathbf{E}_{0}, \mathbf{r}\right), \\
\mathbf{r} \in V_{1}, a^{(2)} \leq r \leq a^{(1)}, \\
\mathbf{E}_{1}=\left(-\boldsymbol{\beta}^{(1)}+\varepsilon_{1}^{-1}\left[-3^{-1} \varepsilon_{1}^{-1}+\left(a^{(2)} / r\right)^{3} \mathbf{I}+\left(a^{(2)}\right)^{3}\right.\right. \\
\left.\left.\times r^{-5}(\mathbf{r} \otimes \mathbf{r})\right] \boldsymbol{\alpha}^{(1)}\right) \mathbf{E}_{0},
\end{gathered}
$$

потенциал и напряженность электрического поля в матрице и ядре включения - соответствующими выражениями из $(65)$ и (66) с учетом того, что $\lambda_{20}, \boldsymbol{\alpha}, \boldsymbol{\alpha}^{(1)}, \boldsymbol{\beta}^{(1)}$ определяются из (73).

2. Для случая анизотропного шара с изотропной сферической оболочкой в изотропной матрице преобразование (7) становится изотропным сжатием

$$
\mathbf{T}_{m}=\sqrt{\varepsilon_{m}} \mathbf{I} .
$$

где $\varepsilon_{m}-$ скалярная диэлектрическая проницаемость матрицы, при этом существенно упростятся выражения $(67)-(71)$ :

$$
a_{k^{\prime}}^{(i)}=\left(\varepsilon_{m}\right)^{-1 / 2} a^{(i)}, \quad k^{\prime}=1^{\prime}, 2^{\prime}, 3^{\prime} ; i=1,2 .
$$

В случае вырождения поверхности-эллипсоида $S_{1}$ в сферу для координат $\xi^{\prime}, \eta^{\prime}, \xi^{\prime}$ определяемой формулами (31) получается предельные выражения:

$$
\begin{gathered}
\xi^{\prime} \rightarrow r^{\prime 2}-\left(a^{\prime(1)}\right)^{2}=\varepsilon_{m}^{-1}\left(r^{2}-\left(a^{(1)}\right)^{2}\right), \\
\eta^{\prime}, \zeta^{\prime} \rightarrow-\left(a^{\prime(1)}\right)^{2}=-\varepsilon_{m}^{-1}\left(a^{(1)}\right)^{2},
\end{gathered}
$$

поэтому для тензорной функции $\mathbf{N}^{\prime}\left(\xi^{\prime}\right)$ имеем аналогично (72)

$$
\mathbf{N}^{\prime}\left(\xi^{\prime}\right)=3^{-1}\left(a^{(1)} / r\right)^{3} \mathbf{I}
$$

откуда

$$
\mathbf{N}_{0}^{\prime}\left(\xi^{\prime}\right)=3^{-1} \varepsilon_{m}^{-1}\left(a^{(1)} / r\right)^{3} \mathbf{I}, \quad \mathbf{L}_{0}^{\prime(1)}=3^{-1} \varepsilon_{m}^{-1} \mathbf{I} .
$$

Также имеем

$R_{\xi^{\prime}}^{\prime(1)}=\varepsilon_{m}^{-3 / 2} r^{3}, \quad h_{1}^{\prime}=1 /(2 r), \quad\left(\mathbf{r}_{\xi^{\prime}}^{\prime} \otimes \mathbf{r}_{\xi^{\prime}}^{\prime}\right)_{0}=4^{-1} r^{-4} \mathbf{r} \otimes \mathbf{r}$.

Выражения для тензоров $\boldsymbol{\lambda}_{20}, \boldsymbol{\alpha}, \boldsymbol{\alpha}^{(1)}, \boldsymbol{\beta}^{(1)}$ принимают вид

$$
\begin{gathered}
\lambda_{20}=9 \varepsilon_{m} \varepsilon_{1}\left[\left(1-v^{\prime}\right)\left(2 \varepsilon_{m}+\varepsilon_{1}\right)\left(\varepsilon_{2}-\varepsilon_{1} \mathbf{I}\right)\right. \\
\left.+3 \varepsilon_{1}\left(2 \varepsilon_{m}+\varepsilon_{1}+v^{\prime}\left(\boldsymbol{\varepsilon}_{2}-\varepsilon_{1} \mathbf{I}\right)\right)\right]^{-1} \\
\boldsymbol{\alpha}=9^{-1} \varepsilon_{m}^{-3 / 2} \varepsilon_{1}^{-1}\left(a^{(1)}\right)^{3}\left[\left(\varepsilon_{1}-\varepsilon_{m}\right)\left(2 \varepsilon_{1} \mathbf{I}+\boldsymbol{\varepsilon}_{2}\right)\right. \\
\left.+v^{\prime}\left(2 \varepsilon_{1}+\varepsilon_{m}\right)\left(\boldsymbol{\varepsilon}_{2}-\varepsilon_{1} \mathbf{I}\right)\right] \boldsymbol{\lambda}_{20}, \\
\boldsymbol{\alpha}^{(1)}\left(\boldsymbol{\varepsilon}_{2}-\varepsilon_{1} \mathbf{I}\right) \boldsymbol{\lambda}_{20}, \quad \boldsymbol{\beta}^{(1)}=-3^{-1} \varepsilon_{1}^{-1}\left(2 \varepsilon_{1} \mathbf{I}+\boldsymbol{\varepsilon}_{2}\right) \boldsymbol{\lambda}_{20} .
\end{gathered}
$$

Потенциал и напряженность электрического поля в сферической оболочке задаются формулами (74) и (75) соответственно, потенциал и напряженность в ядре включения - соответствующими формулами из (65) и (66) с учетом того, что $\lambda_{20}$ определяется из (76), а потенциал и напряженность в матрице - следующими выражениями:

$$
\begin{gathered}
\varphi_{m}(\mathbf{r})=\left(\left(-\mathbf{I}+\varepsilon_{m}^{1 / 2} r^{-3} \boldsymbol{\alpha}\right) \mathbf{E}_{0}, \mathbf{r}\right), \quad \mathbf{r} \in V_{m}, \\
\mathbf{E}_{m}=\left(\mathbf{I}+3\left[-3^{-1} \varepsilon_{m}^{1 / 2} r^{-3} \mathbf{I}+\varepsilon_{m}^{3 / 2} r^{-5}(\mathbf{r} \otimes \mathbf{r})\right] \boldsymbol{\alpha}\right) \mathbf{E}_{0} .
\end{gathered}
$$

Для тензора поляризуемости в соответствии с (50) имеем формулу

$$
\begin{aligned}
\tilde{\boldsymbol{\alpha}}= & \left(a^{(1)}\right)^{3} \varepsilon_{m}\left[\left(\varepsilon_{1}-\varepsilon_{m}\right)\left(2 \varepsilon_{1} \mathbf{I}+\varepsilon_{2}\right)+v^{\prime}\left(2 \varepsilon_{1}+\varepsilon_{m}\right)\right. \\
& \left.\times\left(\varepsilon_{2}-\varepsilon_{1} \mathbf{I}\right)\right]\left[\left(1-v^{\prime}\right)\left(2 \varepsilon_{m}+\varepsilon_{1}\right)\left(\varepsilon_{2}-\varepsilon_{1} \mathbf{I}\right)\right. \\
& \left.+3 \varepsilon_{1}\left(2 \varepsilon_{m}+\varepsilon_{1}+v^{\prime}\left(\varepsilon_{2}-\varepsilon_{1} \mathbf{I}\right)\right)\right]^{-1}
\end{aligned}
$$

являющуюся обобщением известного результата $[8,9]$ на включение с анизотропным ядром.

3. Рассмотрим один из предельных случаев, когда задача (1)-(6) сводится к задаче для однородного эллипсоидального включения в анизотропной среде. Пусть $\varepsilon_{1}=\varepsilon_{2}$, т.е. оболочка и ядро сливаются. Тогда формулы (43) принимают вид

$$
\lambda_{20}=\left(\mathbf{I}+\mathbf{L}_{0}^{\prime(1)}\left(\varepsilon_{1}-\varepsilon_{m}\right)\right)^{-1},
$$




$$
\begin{gathered}
\boldsymbol{\alpha}=3^{-1} \bar{a}^{\prime(1)}\left(\boldsymbol{\varepsilon}_{1}-\boldsymbol{\varepsilon}_{m}\right)\left(\mathbf{I}+\mathbf{L}_{0}^{\prime(1)}\left(\boldsymbol{\varepsilon}_{1}-\boldsymbol{\varepsilon}_{m}\right)\right)^{-1}, \\
\boldsymbol{\alpha}^{(1)}=0, \quad \boldsymbol{\beta}^{(1)}=-\left(\mathbf{I}+\mathbf{L}_{0}^{\prime(1)}\left(\boldsymbol{\varepsilon}_{1}-\boldsymbol{\varepsilon}_{m}\right)\right)^{-1} .
\end{gathered}
$$

Для потенциала, согласно (44), с учетом (77) имеем

$$
\begin{gathered}
\varphi_{m}(\mathbf{r})=\left(\left[-\mathbf{I}+\mathbf{N}_{0}^{\prime}\left(\xi^{\prime}\right)\left(\varepsilon_{1}-\boldsymbol{\varepsilon}_{m}\right)\right.\right. \\
\left.\left.\times\left(\mathbf{I}+\mathbf{L}_{0}^{\prime(1)}\left(\boldsymbol{\varepsilon}_{1}-\boldsymbol{\varepsilon}_{m}\right)\right)^{-1}\right] \mathbf{E}_{0}, \mathbf{r}\right), \\
\mathbf{r} \in V_{m}, \quad \xi^{\prime} \geq 0, \\
\varphi_{1}(\mathbf{r})=\left(-\left(\mathbf{I}+\mathbf{L}_{0}^{\prime(1)}\left(\boldsymbol{\varepsilon}_{1}-\boldsymbol{\varepsilon}_{m}\right)\right)^{-1} \mathbf{E}_{0}, \mathbf{r}\right), \quad \mathbf{r} \in V_{1}, \\
\varphi_{2}(\mathbf{r})=\left(-\left(\mathbf{I}+\mathbf{L}_{0}^{\prime(1)}\left(\boldsymbol{\varepsilon}_{1}-\boldsymbol{\varepsilon}_{m}\right)\right)^{-1} \mathbf{E}_{0}, \mathbf{r}\right), \quad \mathbf{r} \in V_{2} .
\end{gathered}
$$

Напряженность электрического поля $\mathbf{E}_{m}$ в матрице выражается первой из формул (45), а напряженности поля в оболочке и ядре постоянны и равны друг другу:

$$
\mathbf{E}_{1}=\mathbf{E}_{2}=\left(\mathbf{I}+\mathbf{L}_{0}^{\prime(1)}\left(\boldsymbol{\varepsilon}_{1}-\boldsymbol{\varepsilon}_{m}\right)\right)^{-1} \mathbf{E}_{0} .
$$

Выражение (78) совпадает с формулой для напряженности поля внутри эллипсоида с поверхностью $S_{1}$, помещенного в анизотропную среду с приложенным постоянным полем, полученной в [15]. Покажем, что выражения (78) и (45) для напряженности поля внутри эллипсоида и в матрице равносильны следующим выражениям, полученным в [7]:

$$
\begin{gathered}
\mathbf{E}_{1}=\left(\mathbf{I}-\mathbf{S}\left(\mathbf{I}-\boldsymbol{\varepsilon}_{m}^{-1} \boldsymbol{\varepsilon}_{1}\right)^{-1} \mathbf{E}_{\mathbf{0}},\right. \\
\mathbf{E}_{m}=\left(\mathbf{I}+\mathbf{S}^{\infty}(\mathbf{r})\left[\left(\mathbf{I}-\boldsymbol{\varepsilon}_{m}^{-1} \boldsymbol{\varepsilon}_{1}\right)^{-1}-\mathbf{S}\right]^{-1}\right) \mathbf{E}_{0},
\end{gathered}
$$

где $\mathbf{S}$ и $\mathbf{S}^{\infty}(\mathbf{r})-$ внутренний и внешний электрические тензоры Эшелби:

$$
\begin{gathered}
\mathbf{S}=\frac{\operatorname{det}(\mathbf{a})}{2} \int_{0}^{+\infty} \frac{\left[\mathbf{a}^{2}+s \boldsymbol{\varepsilon}_{m}\right]^{-1} \boldsymbol{\varepsilon}_{m} d s}{\sqrt{\operatorname{det}\left(\mathbf{a}^{2}+s \boldsymbol{\varepsilon}_{m}\right)}}, \\
\mathbf{S}^{\infty}(\mathbf{r})=\frac{\operatorname{det}(\mathbf{a})}{2} \int_{\xi^{\prime}}^{+\infty} \frac{\left[\mathbf{a}^{2}+s \boldsymbol{\varepsilon}_{m}\right]^{-1} \boldsymbol{\varepsilon}_{m} d s}{\sqrt{\operatorname{det}\left(\mathbf{a}^{2}+s \boldsymbol{\varepsilon}_{m}\right)}}-\frac{\operatorname{det}(\mathbf{a})}{\sqrt{\operatorname{det}\left(\mathbf{a}^{2}+s \boldsymbol{\varepsilon}_{m}\right)}} \\
\times \frac{\left[\left(\mathbf{a}^{2}+\xi^{\prime} \boldsymbol{\varepsilon}_{m}\right)^{-1} \mathbf{r}\right]\left[\boldsymbol{\varepsilon}_{m}\left(\mathbf{a}^{2}+\xi^{\prime} \boldsymbol{\varepsilon}_{m}\right)^{-1} \mathbf{r}\right]^{T}}{\left[\left(\mathbf{a}^{2}+\xi^{\prime} \boldsymbol{\varepsilon}_{m}\right)^{-1} \mathbf{r}\right]^{T}\left[\boldsymbol{\varepsilon}_{m}\left(\mathbf{a}^{2}+\xi^{\prime} \boldsymbol{\varepsilon}_{m}\right)^{-1} \mathbf{r}\right]},
\end{gathered}
$$

где $\mathbf{a}-$ тензор, диагональный в системе главных осей эллипсоида с поверхностью $S_{1}$, главными компонентами которого являются полуоси поверхности $S_{1} a_{k}^{(1)}$, $k=1,2,3$; величина $\xi^{\prime}$ неявным образом определяется из уравнения

$$
\mathbf{r}^{T}\left(\mathbf{a}^{2}+\xi^{\prime} \boldsymbol{\varepsilon}_{m}\right)^{-1} \mathbf{r}=1 .
$$

Тензор $\mathbf{a}^{2}-$ обратный к тензору $\mathbf{S}_{1}$ квадратичной формы поверхности-эллипсоида $S_{1}$, поэтому он преобразуется как дважды контравариантный при замене системы координат, и после преобразования координат (7) он будет иметь диагональный вид

$$
\mathbf{a}^{\prime 2}=\mathbf{T}_{m}^{-1} \mathbf{a}^{2}\left(\mathbf{T}_{m}^{-1}\right)^{T}=\left(\begin{array}{ccc}
\left(a_{1^{\prime}}^{(1)}\right)^{2} & 0 & 0 \\
0 & \left(a_{2^{\prime}}^{(1)}\right)^{2} & 0 \\
0 & 0 & \left(a_{3^{\prime}}^{(1)}\right)^{2}
\end{array}\right),
$$

так как оси системы координат $x^{1^{\prime}} x^{2^{\prime}} x^{3^{\prime}}$ направлены вдоль осей поверхности-эллипсоида $S_{1}^{\prime}$. В системе $x^{1^{\prime}} x^{2^{\prime}} x^{3^{\prime}}$ уравнение (81) выглядит следующим образом:

$$
\mathbf{r}^{\prime T}\left(\mathbf{a}^{\prime 2}+\xi^{\prime} \mathbf{I}\right)^{-1} \mathbf{r}^{\prime}=1
$$

откуда следует, что оно есть уравнение поверхностиэллипсоида, софокусной с $S_{1}^{\prime}$, а $\xi^{\prime}-$ это первая из эллипсоидальных координат $\xi^{\prime}, \eta^{\prime}, \xi^{\prime}$, вводимых формулами (31). Преобразуем первое слагаемое в выражении (80) для $\mathbf{S}^{\infty}(\mathbf{r})$ :

$$
\begin{aligned}
& \frac{\operatorname{det}(\mathbf{a})}{2} \int_{\xi^{\prime}}^{+\infty} \frac{\left[\mathbf{a}^{2}+s \boldsymbol{\varepsilon}_{m}\right]^{-1} \boldsymbol{\varepsilon}_{m} d s}{\sqrt{\operatorname{det}\left(\mathbf{a}^{2}+s \boldsymbol{\varepsilon}_{m}\right)}}=\frac{\sqrt{\operatorname{det}\left(\mathbf{T}_{m} \mathbf{a}^{\prime 2} \mathbf{T}_{m}^{T}\right)}}{2} \\
& \times \int_{\xi^{\prime}}^{+\infty} \frac{\left[\mathbf{T}_{m}\left(\mathbf{a}^{\prime 2}+s \mathbf{I}\right) \mathbf{T}_{m}^{T}\right]^{-1} d s}{\sqrt{\operatorname{det}\left(\mathbf{T}_{m}\left(\mathbf{a}^{\prime 2}+s \mathbf{I}\right) \mathbf{T}_{m}^{T}\right)}} \boldsymbol{\varepsilon}_{m}=\left(\mathbf{T}_{m}^{T}\right)^{-1} \frac{\operatorname{det}\left(\mathbf{a}^{\prime}\right)}{2} \\
& \quad \times \int_{\xi^{\prime}}^{+\infty} \frac{\left[\mathbf{a}^{\prime 2}+s \mathbf{I}\right]^{-1} d s}{\sqrt{\left.\operatorname{det}\left(\mathbf{a}^{\prime 2}+s \mathbf{I}\right]\right)}} \mathbf{T}_{m}^{-1} \boldsymbol{\varepsilon}_{m}=\left(\mathbf{T}_{m}^{T}\right)^{-1} \mathbf{N}^{\prime}\left(\xi^{\prime}\right) \mathbf{T}_{m}^{-1} \boldsymbol{\varepsilon}_{m} \\
& =\mathbf{N}_{0}^{\prime}\left(\xi^{\prime}\right) \boldsymbol{\varepsilon}_{m},
\end{aligned}
$$

где $\mathbf{N}^{\prime}\left(\xi^{\prime}\right)$ определяется формулами (32) - (34), $\mathbf{N}_{0}^{\prime}\left(\xi^{\prime}\right)-$ формулой (46). С помощью аналогичных преобразований можно показать, что второе слагаемое в выражении для $\mathbf{S}^{\infty}(\mathbf{r})$ приводится к следующей форме:

$$
-\operatorname{det}\left(\mathbf{a}^{\prime}\right) h_{1}^{\prime-2}\left(R_{\xi^{\prime}}^{\prime(1)}\right)^{-1}\left(\mathbf{r}_{\xi^{\prime}}^{\prime} \otimes \mathbf{r}_{\xi^{\prime}}^{\prime}\right)_{0} \boldsymbol{\varepsilon}_{m},
$$

поэтому для $\mathbf{S}^{\infty}(\mathbf{r})$ в итоге имеем выражение

$$
\mathbf{S}^{\infty}(\mathbf{r})=\left[\mathbf{N}_{0}^{\prime}\left(\xi^{\prime}\right)-\operatorname{det}\left(\mathbf{a}^{\prime}\right) h_{1}^{\prime-2}\left(R_{\xi^{\prime}}^{\prime(1)}\right)^{-1}\left(\mathbf{r}_{\xi^{\prime}}^{\prime} \otimes \mathbf{r}_{\xi^{\prime}}^{\prime}\right)_{0}\right] \boldsymbol{\varepsilon}_{m} .
$$

Поскольку $\mathbf{L}_{0}^{\prime(1)}=\mathbf{N}_{0}^{\prime}(0)$, из (80), (82) сразу вытекает, что

$$
\mathbf{S}=\mathbf{L}_{0}^{\prime(1)} \boldsymbol{\varepsilon}_{m} .
$$

Подставляя (83), (84) в (79), после элементарных преобразований получим выражения, в точности совпадающие с (78) и (45). В [8] приведено выражение для напряженности электрического поля внутри эллипсоида в анизотропной матрице, равносильное (79), (80), но немного отличающееся по форме.

Замечание. Из (82) следует, что тензоры $\mathbf{N}_{0}^{\prime}\left(\xi^{\prime}\right)$ и $\mathbf{N}_{00}^{\prime \prime}\left(\xi^{\prime \prime}\right)$, входящие в выражения (44), (45), могут быть записаны в виде

$$
\mathbf{N}_{0}^{\prime}\left(\xi^{\prime}\right)=\frac{\operatorname{det}\left(\mathbf{a}^{(1)}\right)}{2} \int_{\xi^{\prime}}^{+\infty} \frac{\left[\left(\mathbf{a}^{(1)}\right)^{2}+s \boldsymbol{\varepsilon}_{m}\right]^{-1} d s}{\sqrt{\operatorname{det}\left(\left(\mathbf{a}^{(1)}\right)^{2}+s \boldsymbol{\varepsilon}_{m}\right)}},
$$




$$
\mathbf{N}_{00}^{\prime \prime}\left(\xi^{\prime \prime}\right)=\frac{\operatorname{det}\left(\mathbf{a}^{(2)}\right)}{2} \int_{\xi^{\prime \prime}}^{+\infty} \frac{\left[\left(\mathbf{a}^{(2)}\right)^{2}+s \boldsymbol{\varepsilon}_{1}\right]^{-1} d s}{\sqrt{\operatorname{det}\left(\left(\mathbf{a}^{(2)}\right)^{2}+s \boldsymbol{\varepsilon}_{1}\right)}},
$$

где $\mathbf{a}^{(1)}, \mathbf{a}^{(2)}$ - тензоры с главными компонентами, равными полуосям поверхностей $S_{1}, S_{2}$, а главные оси совпадают с их осями соответственно.

\section{Заключение}

Основными результатами настоящей работы являются аналитические выражения для потенциала и напряженности электрического поля в анизотропных матрице, оболочке и ядре включения, представленные формулами (44) и (45) соответственно, выражение для тензора поляризуемости включения - формула (51). Также получены выражения для потенциала и напряженности поля в частном случае задачи, когда оболочка включения - изотропная (формулы (61), (62) и (58) соответственно).

Результаты, полученные в работе, могут быть использованы при решении задач оценки эффективных характеристик неоднородных сред, например, поликристаллов с учетом межзеренного пространства и композитов с включениями вложенной структуры, а также для учета граничного слоя между включениями и матрицей.

Работа выполнена при финансовой поддержке Российского фонда фундаментальных исследований (гранты № 16-08-00262-а и 17-08-01374-a).

\section{Список литературы}

[1] Stroud D. // Phys. Rev. B. 1975. Vol. 12. N 8. P. 3368-3373.

[2] Фокин А.Г. // УФН. 1996. Т. 166 № 10. С. 1069-1093. [Fokin A.G. // Phys. Usp. 1996. Vol. 39. P. 1009-1032.]

[3] Bruggeman D.A.G. // Ann. Phys. 1935. Band 24. P. 636-664.

[4] Апресян Л.А., Власов Д.В., Задорин Д.А., Красовский В.И. // ЖТФ. 2017. Т. 87. Вып. 1. С. 1017. DOI: $10.21883 /$ JTF.2017.01.44011.1841 [Apresyan L.A., Vlasov D.V., Zadorin D.A., Krasovskii V.I. // Tech. Phys. 2017. Vol. 62. N 1. P. 6-13.]

[5] Колесников В.И., Бардушкин В.В., Лавров И.В., Сычев А.П., Яковлев В.Б. // ДАН. 2017. Т. 476. № 3. С. 280 284. DOI: 10.7868/S0869565217270081 [Kolesnikov V.I., Bardushkin V.V., Lavrov I.V., Sychev A.P., Yakovlev V.B. // Dokl. Phys. 2017. Vol. 62. N 9. P. 415-419.] DOI: $10.1134 / \mathrm{S} 1028335817090087]$

[6] Лавров И.В., Яковлев В.Б. // ЖТФ. 2017. Т. 87. Вып. 7. С. 963-972. DOI: 10.21883/JTF.2017.07.44663.1964 [Lavrov I.V., Yakovlev V.B. // Tech. Phys. 2017. Vol. 62. N 7. P. 979-988.]

[7] Giordano S., Palla P.L. // J. Phys. A: Math. Theor. 2008. Vol. 41. P. 415205. DOI:10.1088/1751-8113/41/41/415205

[8] Sihvola A. Electromagnetic Mixing Formulas and Applications. The Institution of Electrical Engineers, London, 1999.
[9] Борен К., Хафмен Д. Поглощение и рассеяние света малыми частицами. М.: Мир, 1986. 660 c. [Bohren C., Huffman D.R. Absorption and Scattering of Light by Small Particles. Wiley, NY., 1983.]

[10] Ландау Л.Д., Лифиии, Е.М. Теоретическая физика. Т. 8. Электродинамика сплошных сред. М.: Наука, 1992. 664 с. [Landau L.D., Lifshitz E.M. Electrodynamics of Continuous Media. Butterworth-Heinemann, 1984.]

[11] Стрэттон Джс. Теория электромагнетизма. М.-Л.: ГИТТЛ, 1948. 539 с. [Stratton J.A. Electromagnetic Theory. McGraw-Hill, 1941.]

[12] Апресян Л.А., Власов Д.В. // ЖТФ. 2014. Т. 84. Вып. 12. C. 23-28. [Apresyan L.A., Vlasov D.V. // Tech. Phys. 2014. Vol. 59. N 12. P. 1760-1765.]

[13] Лерман Л.Б. // Химия, физика и технология поверхности. 2008. Вып. 14. С. 91-100.

[14] Ораевский А.А., Ораевский А.Н. // Квантовая электроника. 2002. Т. 32. № 1. С. 79-82. [Oraevsky A.A., Oraevsky A.N. // Quant. Electr. 2002. Vol. 32. N 1. P. 79-82.]

[15] Лавров И.В. // Фундам. пробл. радиоэлектрон. приборостроения. 2013. Т. 13. № 1. С. 44-47. 